\title{
ASYMPTOTICS OF BOND YIELDS AND VOLATILITIES FOR EXTENDED VASICEK MODELS UNDER THE REAL-WORLD MEASURE
}

\author{
K. FERGUSSON
}

\begin{abstract}
Vasicek's short rate model is a mean reverting model of the short rate which permits closed-form pricing formulae of zero coupon bonds and options on zero coupon bonds. This article supplies proofs which are valid for any single factor mean reverting Gaussian short rate model having timeinhomogeneous parameters. The formulae are for the expected present value of payoffs under the real-world probability measure, known as actuarial pricing. Importantly, we give formulae for asymptotic levels of bond yields and volatilities for extended Vasicek models when suitable conditions are imposed on the model parameters.
\end{abstract}

\section{INTRODUCTION}

In actuarial science the short term interest rate plays a central role in valuations of future cashflows, particularly those pertaining to short-tail insurance policies. A short rate model is a mathematical model of the instantaneous, continuously compounded deposit rate for a specific currency. The most realistic proxy for the short rate among investible securities is probably the overnight cash deposit rate, expressed as a continuously compounded rate. Short rates are typically modelled as stochastic processes and coverages of short rate models can be found, for example, in Rebonato [1998] and Brigo and Mercurio [2006].

The short rate models considered in this paper are called Gaussian short rate models and are specified by stochastic differential equations (SDEs) with a single noise source and with time-dependent coefficients. From an actuarial pricing perspective the availability of explicit pricing formulae for calculations involving the short term interest rate is of extreme importance. The class of short rate dynamics where one can probably expect the widest range of explicit valuation formulae is probably the Gaussian class. They are convenient and also reasonably realistic for pricing future cash flows and contingent claims. They have explicit closed-form formulae for their transition density functions and also allow negative values. Of particular importance for actuaries is the requirement that the long-term bond yield implied by the model be a finite constant, which is guaranteed for the Vasicek model

Date: February 28, 2017.

1991 Mathematics Subject Classification. Primary 62P05; Secondary 60G35, 62P20.

Key words and phrases. Actuarial pricing, stochastic short rate, Gaussian short rate, Vasicek model, Hull-White model.

The author is grateful for the referee's helpful comments and suggestions and for Professor Eckhard Platen's proofreading and support. This research is supported by an Australian Government Research Training Program Scholarship. 
Figure 1. Comparison of empirical probability density function of annual change in short rate with that of the fitted normal distribution (US $1 Y$ cash rates 1871 - 2010).

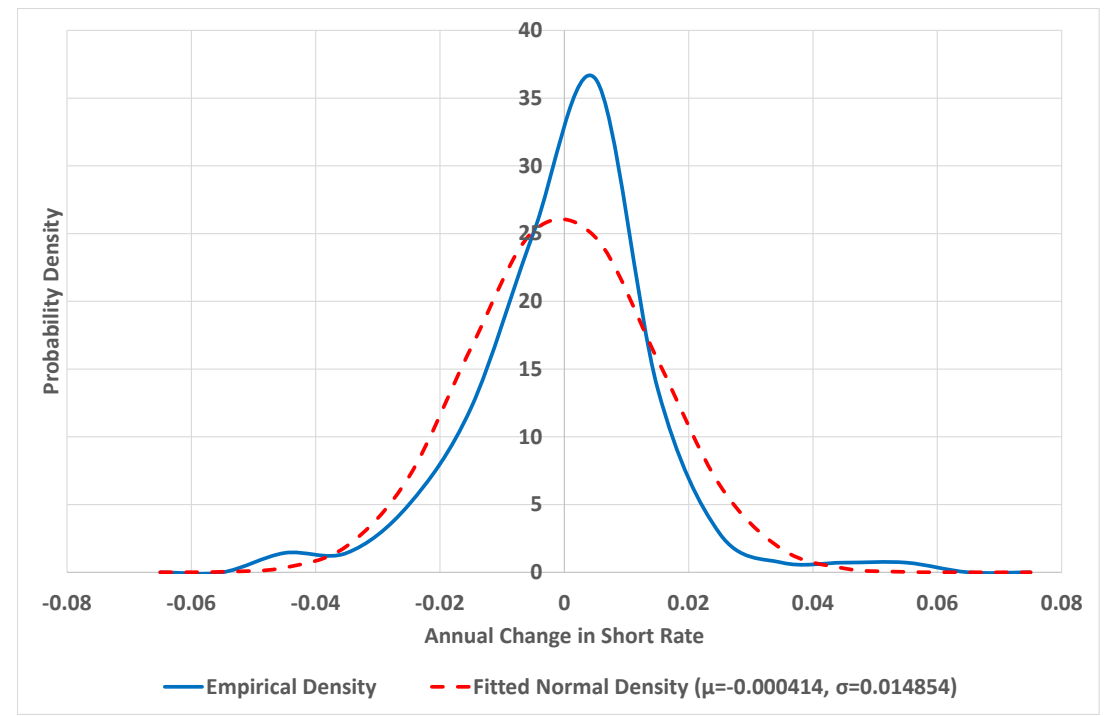

but not necessarily for extended Vasicek models. Figure 1 illustrates the asymmetry of the distribution of annual changes in the short rate for US cash rates, which corresponds to the leverage effect in bond markets. While this effect is not captured by extended Vasicek models, it is a short-term effect which is less pronounced when analysing the asymptotic behaviour of bond yields and volatilities.

A particular example of a Gaussian short rate model is the well-known Vasicek model, which is a linear mean reverting stochastic model, see Vasicek [1977]. This ensures that interest rates adhere to a long run reference level.

Working on a filtered probability space $\left(\Omega, \underline{\mathcal{A}},\left(\mathcal{A}_{t}\right)_{t \geq 0}, P\right)$, the SDE for the extended Vasicek short rate model or Hull-White extension is given as

$$
d r_{t}=\kappa_{t}\left(\bar{r}_{t}-r_{t}\right) d t+\sigma_{t} d Z_{t}
$$

where $r_{t}$ is the short rate at time $t \geq 0, Z_{t}$ is a Wiener process adapted to the filtration $\left(\mathcal{A}_{t}\right)_{t \geq 0}$ and $\bar{r}, \kappa$ and $\sigma$ are positive deterministic functions of time. The aim of this paper is to provide for this type of model a wide range of valuation formulae that are useful in actuarial valuations and, for suitable conditions on the model parameters, to show that the implied long-term bond yield is finite.

In Section 2 we introduce actuarial pricing and in Section 3 we describe the Vasicek short rate model and the Hull-White extension, the explicit solutions of which are given in Section 4. We provide formulae for the transition density function in Sections 5 and 6, the zero coupon bond (ZCB) price in Section 7 , bond yields and instantaneous forward rates in Section 8 and the price of an option on the ZCB 
in Section 9. We go deliberately through all steps of the derivations, even though some of these may be well-known under risk neutral assumptions. Note, however, our pricing will be done under the real-world probability measure $P$ and not under some assumed risk neutral measure.

\section{Actuarial Pricing}

Let

$$
B_{t}=B_{0} \exp \left\{\int_{0}^{t} r_{s} d s\right\}
$$

denote for $t \geq 0$ the savings account, the locally risk-free asset. Actuarial pricing, at the present time $t$, of a contingent claim $H_{T}$ occurring at some future time $T$ is computed using the actuarial pricing formula

$$
V_{t}=E\left(\frac{B_{t}}{B_{T}} H_{T} \mid \mathcal{A}_{t}\right)
$$

where $E\left(\cdot \mid \mathcal{A}_{t}\right)$ denotes conditional expectation under the real-world probability measure $P$. It is the expected present value of the contingent claim $H_{T}$ occurring at time $T$. Under the classical risk neutral assumptions every discounted price process, discounted by the savings account, is forming a martingale under some risk neutral probability measure $Q$. The above actuarial pricing formula arises from the risk neutral pricing formula

$$
V_{t}^{*}=E^{Q}\left(\frac{B_{t}}{B_{T}} H_{T} \mid \mathcal{A}_{t}\right)
$$

when the random variable

$$
\frac{B_{t}}{B_{T}} H_{T}
$$

is independent from the Radon-Nikodym derivative $\left.\frac{d Q}{d P}\right|_{\mathcal{A}_{T}}$, where $Q$ is an assumed risk neutral measure, equivalent to the real-world probability measure $P$. For most models considered in the literature, such an equivalent risk neutral measure is assumed to exist, but for more general models this may not be the case.

To cover such cases, pricing under the real-world probability measure has been formalised with Platen's benchmark approach, described in Platen [2002] and Platen and Heath [2006], and the above actuarial pricing formula emerges also as a special case under this approach. In particular, the benchmark approach allows one to apply formally risk neutral pricing even when as equivalent risk neutral probability measure does not exist, see Platen and Taylor [2016]. Therefore, the pricing formulae developed in this paper can be applied more widely than typically believed.

From the actuarial pricing formula (2.2) it follows in the case when the contingent claim is independent from the short rate that one has

$$
V_{t}=E\left(\frac{B_{t}}{B_{T}} \mid \mathcal{A}_{t}\right) E\left(H_{T} \mid \mathcal{A}_{t}\right)=G_{T}(t) E\left(H_{T} \mid \mathcal{A}_{t}\right)
$$

for $t \in[0, T]$. Here $G_{T}(t)$ is the price of a ZCB maturing at time $T$, and we have the actuarial price $G_{T}(t)$ at time $t \in[0, T]$ as

$$
G_{T}(t)=E\left(\exp \left\{-\int_{t}^{T} r_{s} d s\right\} \mid \mathcal{A}_{t}\right) .
$$


Thus the price of a ZCB plays a central role in actuarial pricing.

For a $\bar{T}$-expiry call option, $0<\bar{T} \leq T<\infty$, on such a ZCB with strike price $K$, the actuarial price $c_{\bar{T}, T, K}(t)$ at time $t \int[0, T]$ is

$$
c_{\bar{T}, T, K}(t)=E\left(\exp \left\{-\int_{t}^{\bar{T}} r_{s} d s\right\}\left(G_{T}(\bar{T})-K\right)^{+} \mid \mathcal{A}_{t}\right) .
$$

Corresponding formulae for a put option, asset-or-nothing call option, asset-ornothing put option, cash-or-nothing call option and cash-or-nothing put option, each on such a ZCB, are

$$
\begin{aligned}
& p_{\bar{T}, T, K}(t)=E\left(\exp \left\{-\int_{t}^{\bar{T}} r_{s} d s\right\}\left(K-G_{T}(\bar{T})\right)^{+} \mid \mathcal{A}_{t}\right) \\
& A_{\bar{T}, T, K}^{+}(t)=E\left(\exp \left\{-\int_{t}^{\bar{T}} r_{s} d s\right\} G_{T}(\bar{T}) \mathbf{1}_{G_{T}(\bar{T})>K} \mid \mathcal{A}_{t}\right) \\
& A_{\bar{T}, T, K}^{-}(t)=E\left(\exp \left\{-\int_{t}^{\bar{T}} r_{s} d s\right\} G_{T}(\bar{T}) \mathbf{1}_{G_{T}(\bar{T}) \leq K} \mid \mathcal{A}_{t}\right) \\
& B_{\bar{T}, T, K}^{+}(t)=E\left(\exp \left\{-\int_{t}^{\bar{T}} r_{s} d s\right\} \mathbf{1}_{G_{T}(\bar{T})>K} \mid \mathcal{A}_{t}\right) \\
& B_{\bar{T}, T, K}^{-}(t)=E\left(\exp \left\{-\int_{t}^{\bar{T}} r_{s} d s\right\} \mathbf{1}_{G_{T}(\bar{T}) \leq K} \mid \mathcal{A}_{t}\right),
\end{aligned}
$$

respectively. Here $\mathbf{1}_{X>K}$ denotes the indicator function, equalling one if the random variable $X$ exceeds the value $K$, and zero otherwise.

The availability of explicit formulae for the transition density function of the short rate makes it not only possible to provide explicit formulae for the above prices but also to fit the model to historical data using maximum likelihood estimation, as demonstrated in Fergusson and Platen [2015]. In determining the formulae for ZCBs and ZCB options we derive also a formula for the moment generating function of $\log \left(B_{\bar{T}} / B_{t}\right)$, which can be used for the approximation of other prices.

\section{Vasicek Short Rate Model and Extensions}

The Vasicek model was proposed in Vasicek [1977], and extended in Hull and White [1990] to the Hull-White model whose drift and diffusion parameters are made time dependent, which also became known as the extended Vasicek model.

This SDE (1.1) is the Ornstein-Uhlenbeck SDE whose explicit solution is obtained by solving the SDE of $q_{t}=r_{t} e_{t}$ with

$$
e_{t}=\exp \left\{\int_{0}^{t} \kappa_{s} d s\right\}
$$

where

$$
d q_{t}=d\left(r_{t} e_{t}\right)=\kappa_{t} e_{t} \bar{r}_{t} d t+e_{t} \sigma_{t} d Z_{t} .
$$

Vasicek's model, which is a special case of (1.1) with $\kappa_{t}, \bar{r}_{t}, \sigma_{t}$ constant, and whose SDE is

$$
d r_{t}=\kappa\left(\bar{r}-r_{t}\right) d t+\sigma d Z_{t},
$$

was probably the first interest rate model to capture mean reversion, an essential characteristic of the interest rate that sets it apart from simpler models. Thus, 
under the real-world probability measure, as opposed to stock prices, for instance, interest rates are not expected to rise indefinitely. This is because at very high levels they would hamper economic activity, prompting a decrease in interest rates. Similarly, interest rates are unlikely to decrease indefinitely. As a result, interest rates move mainly in a range, showing a tendency to revert to a long run value.

The drift factor $\kappa\left(\bar{r}-r_{t}\right)$ represents the expected instantaneous change in the interest rate at time $t$. The parameter $\bar{r}$ represents the long run reference value towards which the interest rate reverts. Indeed, in the absence of uncertainty, the interest rate would remain constant when it has reached $r_{t}=\bar{r}$. The parameter $\kappa$, governing the speed of adjustment, needs to be positive to ensure stability around the long term value. For example, when $r_{t}$ is below $\bar{r}$, the drift term $\kappa\left(\bar{r}-r_{t}\right)$ becomes positive for positive $\kappa$, generating a tendency for the interest rate to move upwards.

The main disadvantage seemed that, under Vasicek's model, it is theoretically possible for the interest rate to become negative. In the previous academic literature this has been interpreted as an undesirable feature. However, on several occasions the market generated in recent years negative interest rates, for example in Switzerland and in Europe. The possiblity of negative interest rates is excluded in the Cox-Ingersoll-Ross model (see Cox et al. [1985]), the exponential Vasicek model (see Brigo and Mercurio [2001]), the model of Black et al. [1990] and the model of Black and Karasinski [1991], among many others. See Brigo and Mercurio [2006] for further discussions.

Another disadvantage is that the Vasicek model does not capture stochastic volatility, evident in the graph of the quadratic variation of the short rate in Figure 2. Therefore, a serious consideration of real-world dynamics would require models whose stochastic differential equations of the short rate have stochastic volatility, such as the Cox-Ingersoll-Ross model and the $3 / 2$ model (see Platen [1999]). However, owing to the mean reverting nature of stochastic volatility, this will have less impact on the asymptotic behaviour of bond volatilities.

The Vasicek model was further extended in the Hull-White model (see Hull and White [1990]), by allowing time dependence in the drift parameters. The HullWhite model is specified by the SDE

$$
d r_{t}=\left\{\theta(t)+a(t)\left(b-r_{t}\right)\right\} d t+\sigma(t) d Z_{t},
$$

where $\theta(t), a(t)$ and $\sigma(t)$ are deterministic functions of $t$, satisfying $a(t)>0$ and $\sigma(t)>0$ and $b$ is a constant. When setting $\kappa_{t}=a(t)$ and $\bar{r}_{t}=b+\theta(t) / a(t)$ in (1.1) we obtain (3.4). Further, in (3.4) when setting $a(t)=0$ and $\sigma(t)$ equal to a positive constant $\sigma$ we obtain

$$
d r_{t}=\theta(t) d t+\sigma d Z_{t}
$$

which is implicitly what is employed in Ho and Lee [1986].

We now provide an explicit solution to each of the SDE (3.3) and the SDE (3.4) from which we determine the associated transition density function.

\section{Explicit Formula for the Short Rate}

An explicit solution to the Ornstein-Uhlenbeck process is straightforwardly obtained in the following theorem. 
FIGURE 2. Quadratic variation of the short rate (US 1Y cash rates $1871-2010)$.

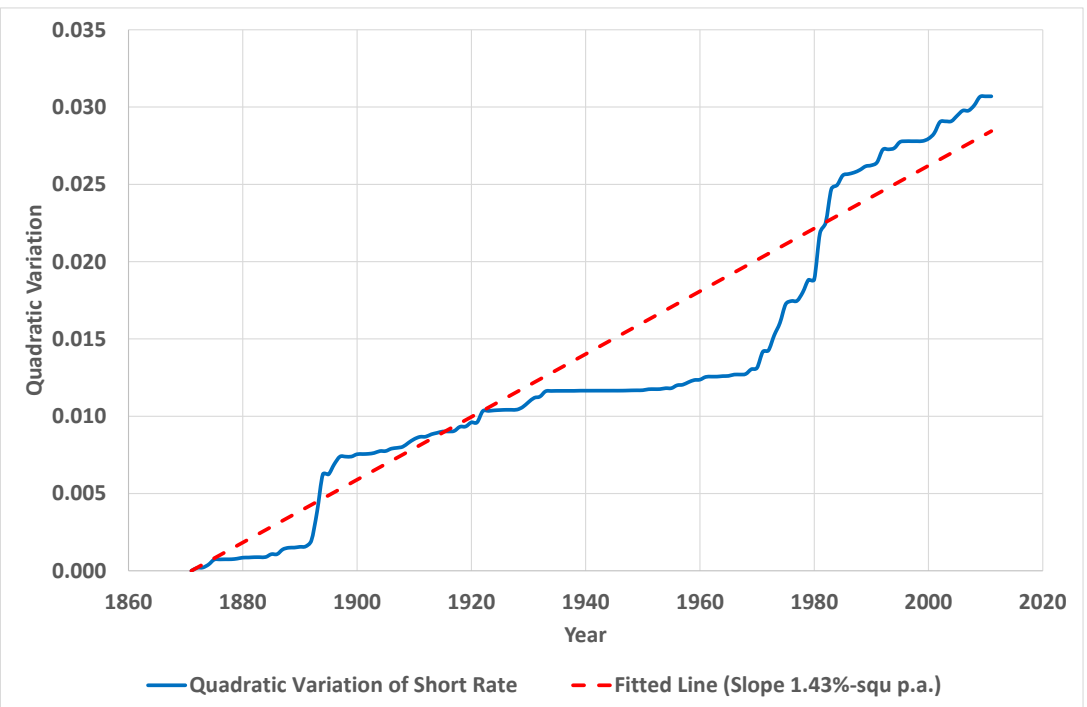

Proposition 1. The short rate $r_{t}$ satisfying the Vasicek SDE (3.3) has solution

$$
r_{t}=r_{s} \exp (-\kappa(t-s))+\bar{r}(1-\exp (-\kappa(t-s)))+\sigma \int_{s}^{t} \exp (-\kappa(t-u)) d Z_{u}
$$

for times $s$ and $t$ with $0 \leq s<t$ and for positive constants $\bar{r}, \kappa$ and $\sigma$. Here $Z$ is the Wiener process in (3.3).

Proof. Integrating both sides of (3.2) between times $s$ and $t$ gives

$$
r_{t} \exp (\kappa t)-r_{s} \exp (\kappa s)=\kappa \bar{r} \int_{s}^{t} \exp (\kappa u) d u+\sigma \int_{s}^{t} \exp (\kappa u) d Z_{u} .
$$

Multiplying both sides by $\exp (-\kappa t)$ and simplifying gives (4.1).

The proof is similar for the solution to the Hull-White SDE in (3.4).

Proposition 2. The short rate $r_{t}$ satisfying the Hull-White SDE (3.4) has solution

$$
\begin{aligned}
r_{t} & =r_{s} \exp \left\{-\int_{s}^{t} a(\tau) d \tau\right\}+\int_{s}^{t} \exp \left\{-\int_{u}^{t} a(\tau) d \tau\right\}\{\theta(u)+a(u) b\} d u \\
& +\int_{s}^{t} \exp \left\{-\int_{u}^{t} a(\tau) d \tau\right\} \sigma(u) d Z_{u}
\end{aligned}
$$

for times $s$ and $t$ with $0 \leq s<t$, for positive functions $\theta(u), a(u)$ and $\sigma(u)$ and for a constant $b$. Here $Z$ is the Wiener process in (3.4). 


\section{Transition Density of the Short Rate}

As is the case for the Ho-Lee model in (3.5) and the Hull-White model in (3.4), the transition density function of the Vasicek short rate is that of a normal distribution.

Corollary 1. For times $s$ and $t$ with $0 \leq s<t \leq T$ the transition density of the short rate $r_{t}$ in (3.3) is given by

$$
\begin{aligned}
p_{r}\left(s, r_{s}, t, r_{t}\right) & =\frac{1}{\sqrt{2 \pi \sigma^{2} \frac{1-\exp (-2 \kappa(t-s))}{2 \kappa}}} \\
& \times \exp \left(-\frac{1}{2}\left(\frac{r_{t}-r_{s} \exp (-\kappa(t-s))-\bar{r}(1-\exp (-\kappa(t-s)))}{\sqrt{\sigma^{2} \frac{1-\exp (-2 \kappa(t-s))}{2 \kappa}}}\right)\right) .
\end{aligned}
$$

Proof. From (4.1) we see that $r_{t}$ conditioned upon $r_{s}$ is normally distributed and has expected value

$$
E\left(r_{t} \mid \mathcal{A}_{s}\right)=r_{s} \exp (-\kappa(t-s))+\bar{r}(1-\exp (-\kappa(t-s)))
$$

and variance

$$
V A R\left(r_{t} \mid \mathcal{A}_{s}\right)=\sigma^{2} \int_{s}^{t} \exp (-2 \kappa(t-u)) d u=\sigma^{2}(1-\exp (-2 \kappa(t-s))) /(2 \kappa)
$$

The transition density function must, therefore, be given by (5.1).

A graph of the transition density function is shown in Figure 3 for parameters shown in (5.4). These are maximum likelihood estimates derived from Shiller's annual data set of one-year US deposit rates from 1871 to 2012, with standard errors shown in brackets.

$$
\begin{aligned}
\bar{r} & =0.042994(0.0080023) \\
\kappa & =0.162953(0.053703) \\
\sigma & =0.015384(0.00099592) .
\end{aligned}
$$

As for other Gaussian short rate models such as the Ho-Lee model and the HullWhite model, a potential disadvantage of the Vasicek model is the possibility of negative interest rates.

We show the parameter estimate for the mean reverting level $\bar{r}$ alongside the historical short rates in Figure 4. We note that for the periods after 1930 a time dependent reference level may be appropriate but we deliberately keep constant parameters in this paper to clarify the methodology.

A lemma, which can be deduced from Corollary 1 and which will be used later, is as follows.

Lemma 1. For the Vasicek process in (3.3) and times $s, t$ with $s \leq t$ let the mean and variance of $r_{t}$ given $r_{s}$ be defined as

$$
\begin{aligned}
m_{s}(t) & =E\left(r_{t} \mid \mathcal{A}_{s}\right) \\
v_{s}(t) & =V A R\left(r_{t} \mid \mathcal{A}_{s}\right)=E\left(\left(r_{t}-m_{s}(t)\right)^{2} \mid \mathcal{A}_{s}\right) .
\end{aligned}
$$


FiguRE 3. Vasicek transition density function of US cash rates based at year 2000 and short rate 0.064 .

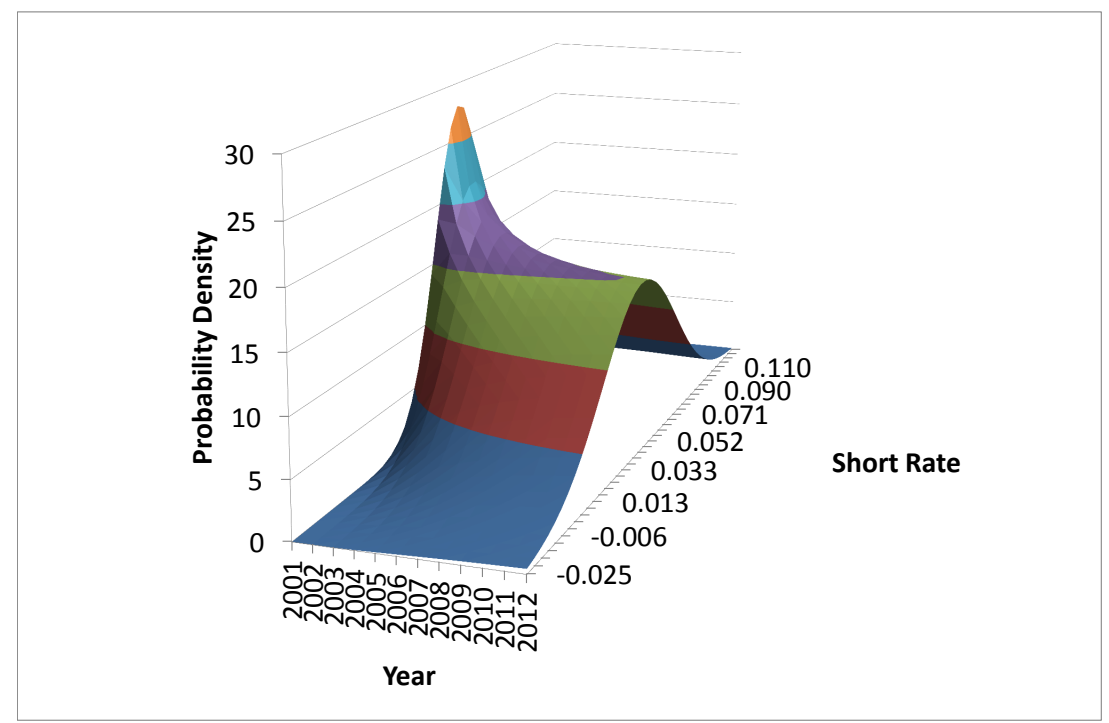

Then we have the explicit formulae

$$
\begin{aligned}
m_{s}(t) & =\bar{r} \kappa B(s, t)+r_{s}(1-\kappa B(s, t)) \\
v_{s}(t) & =\sigma^{2}\left(B(s, t)-\frac{1}{2} \kappa B(s, t)^{2}\right),
\end{aligned}
$$

where

$$
B(s, t)=(1-\exp (-\kappa(t-s))) / \kappa .
$$

Proof. Integrating the SDE (3.3) gives

$$
r_{t}=r_{s}+\int_{s}^{t} \kappa\left(\bar{r}-r_{u}\right) d u+\int_{s}^{t} \sigma d Z_{u}
$$

and taking expectations conditioned on $r_{s}$ gives

$$
m_{s}(t)=r_{s}+\int_{s}^{t} \kappa\left(\bar{r}-m_{s}(u)\right) d u .
$$

This can be written as a first order ordinary differential equation in $m_{s}(t)$

$$
m_{s}(t)^{\prime}=\kappa\left(\bar{r}-m_{s}(t)\right)
$$

with initial condition $m_{s}(s)=r_{s}$, the solution of which is straightforward. Now the SDE of $r_{t}^{2}$ is, by Ito's Lemma,

$$
d r_{t}^{2}=\left(\sigma^{2}+2 \kappa \bar{r} r_{t}-2 \kappa r_{t}^{2}\right) d t+2 \sigma r_{t} d Z_{t}
$$


FiguRe 4. Actual short rate and fitted Vasicek mean reverting level for US cash rates.

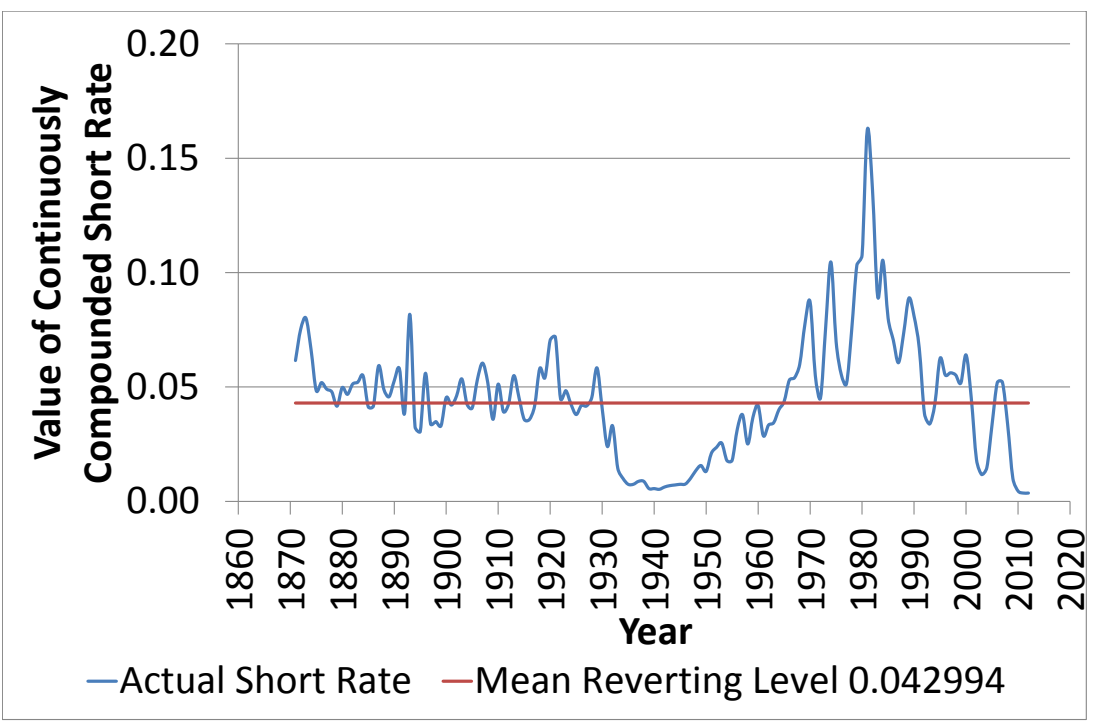

and integrating this SDE gives

$$
r_{t}^{2}=r_{s}^{2}+\int_{s}^{t}\left(\sigma^{2}+2 \kappa \bar{r} r_{u}-2 \kappa r_{u}^{2}\right) d u+\int_{s}^{t} 2 \sigma r_{u} d Z_{u}
$$

Taking expectations conditioned on $r_{s}$, and defining $m_{s}^{(2)}(t)=E\left(r_{t}^{2} \mid \mathcal{A}_{s}\right)$, gives

$$
m_{s}^{(2)}(s)=r_{s}^{2}+\int_{s}^{t}\left(\sigma^{2}+2 \kappa \bar{r} m_{s}(u)-2 \kappa m_{s}^{(2)}(u)\right) d u
$$

from which we have the ordinary differential equation

$$
m_{s}^{(2)^{\prime}}(t)=\sigma^{2}+2 \kappa \bar{r} m_{s}(t)-2 \kappa m_{s}^{(2)}(t) .
$$

Multiplying both sides by $\exp (2 \kappa t)$ and rearranging gives

$$
\begin{aligned}
\frac{d}{d t}\left(\exp (2 \kappa t) m_{t}^{(2)}\right) & =\sigma^{2} \exp (2 \kappa t)+2 \kappa \bar{r} m_{s}(t) \exp (2 \kappa t) \\
& =\sigma^{2} \exp (2 \kappa t)+2 \kappa \bar{r} \exp (2 \kappa t)\left(\kappa \bar{r} B(s, t)+r_{s}(1-\kappa B(s, t))\right) \\
& =\left(\sigma^{2}+2 \kappa \bar{r} r_{s}\right) \exp (2 \kappa t)+2 \kappa^{2} \bar{r}\left(\bar{r}-r_{s}\right) \exp (2 \kappa t) B(s, t) .
\end{aligned}
$$


We note that

$$
\begin{aligned}
\int_{s}^{t} \exp (2 \kappa u) B(s, u) d u & =\frac{1}{2} \exp (2 \kappa t) B(s, t)^{2} \\
\int_{s}^{t} \exp (2 \kappa u) d u & =\frac{1}{2} \exp (2 \kappa t)\left(2 B(s, t)-\kappa B(s, t)^{2}\right) .
\end{aligned}
$$

Therefore, integrating both sides of (5.15) from $s$ to $t$ gives

$$
\begin{aligned}
\exp (2 \kappa t) m_{s}^{(2)}(t) & =\exp (2 \kappa s) r_{t}^{2}+\left(\sigma^{2}+2 \kappa \bar{r} r_{s}\right) \frac{1}{2} \exp (2 \kappa t)\left(2 B(s, t)-\kappa B(s, t)^{2}\right) \\
& +2 \kappa^{2} \bar{r}\left(\bar{r}-r_{s}\right) \frac{1}{2} \exp (2 \kappa t) B(s, t)^{2} \\
& =\exp (2 \kappa s) r_{t}^{2}+\left(\sigma^{2}+2 \kappa \bar{r} r_{s}\right) \exp (2 \kappa t) B(s, t) \\
& +\frac{1}{2} \exp (2 \kappa t) B(s, t)^{2}\left(2 \kappa^{2} \bar{r}\left(\bar{r}-r_{s}\right)-\kappa\left(\sigma^{2}+2 \kappa \bar{r} r_{s}\right)\right) \\
& =\exp (2 \kappa s) r_{t}^{2}+\left(\sigma^{2}+2 \kappa \bar{r} r_{s}\right) \exp (2 \kappa t) B(s, t) \\
& +\frac{1}{2} \exp (2 \kappa t) B(s, t)^{2}\left(2 \kappa^{2} \bar{r}^{2}-\kappa \sigma^{2}-4 \kappa^{2} \bar{r} r_{s}\right)
\end{aligned}
$$

and dividing both sides by $\exp (2 \kappa t)$ gives

$$
\begin{aligned}
m_{s}^{(2)}(t) & =r_{s}^{2} \exp (-2 \kappa(t-s))+\left(\sigma^{2}+2 \kappa \bar{r} r_{s}\right) B(s, t) \\
& +\frac{1}{2} B(s, t)^{2}\left(2 \kappa^{2} \bar{r}^{2}-\kappa \sigma^{2}-4 \kappa^{2} \bar{r} r_{s}\right) .
\end{aligned}
$$

The variance is computed as $v_{s}(t)=m_{s}^{(2)}(t)-\left(m_{s}(t)\right)^{2}$, that is

$$
\begin{aligned}
v_{s}(t) & =r_{s}^{2} \exp (-2 \kappa(t-s))+\left(\sigma^{2}+2 \kappa \bar{r} r_{s}\right) B(s, t) \\
& +\frac{1}{2} B(s, t)^{2}\left(2 \kappa^{2} \bar{r}^{2}-\kappa \sigma^{2}-4 \kappa^{2} \bar{r} r_{s}\right) \\
& -\left(\bar{r} \kappa B(s, t)+r_{s}(1-\kappa B(s, t))\right)^{2} \\
& =r_{s}^{2} \exp (-2 \kappa(t-s))+\left(\sigma^{2}+2 \kappa \bar{r} r_{s}\right) B(s, t) \\
& +\frac{1}{2} B(s, t)^{2}\left(2 \kappa^{2} \bar{r}^{2}-\kappa \sigma^{2}-4 \kappa^{2} \bar{r} r_{s}\right) \\
& -\bar{r}^{2} \kappa^{2} B(s, t)^{2}-r_{s}^{2}(1-\kappa B(s, t))^{2}-2 \kappa \bar{r} r_{s}\left(B(s, t)-\kappa B(s, t)^{2}\right) \\
& =\sigma^{2} B(s, t)-\frac{1}{2} \kappa \sigma^{2} B(s, t)^{2}
\end{aligned}
$$

as required.

For the Hull-White model we have the following corollary. 
Corollary 2. For times $s$ and $t$ with $0 \leq s<t \leq T$ the transition density of the short rate $r_{t}$ in (3.4) is given by

$$
\begin{aligned}
p_{r}\left(s, r_{s}, t, r_{t}\right) & =\frac{1}{\sqrt{2 \pi v_{s}(t)}} \\
& \times \exp \left(-\frac{1}{2}\left(\frac{r_{t}-m_{s}(t)}{\sqrt{v_{s}(t)}}\right)^{2}\right),
\end{aligned}
$$

where

$$
\begin{aligned}
m_{s}(t) & =r_{s} \exp \left\{-\int_{s}^{t} a(\tau) d \tau\right\}+\int_{s}^{t} \exp \left\{-\int_{u}^{t} a(\tau) d \tau\right\}\{\theta(u)+a(u) b\} d u \\
v_{s}(t) & =\int_{s}^{t} \exp \left\{-2 \int_{u}^{t} a(\tau) d \tau\right\} \sigma(u)^{2} d u .
\end{aligned}
$$

\section{The Savings Account and its Transition Density}

The savings account consists of the dollar wealth accumulated continuously at the short rate, given an initial deposit of one dollar at time zero. The value of the savings account at time $t$ is given in (2.1). The following lemma leads to the formula for the savings account value under the Vasicek model.

Lemma 2. Let $r_{t}$ satisfy the Vasicek SDE (3.3). Then

$$
\int_{t}^{\bar{T}} r_{s} d s=r_{t} B(t, \bar{T})+\bar{r}(\bar{T}-t-B(t, \bar{T}))+\sigma \int_{t}^{\bar{T}} B(u, \bar{T}) d Z_{u}
$$

where

$$
B(t, \bar{T})=\frac{1}{\kappa}(1-\exp (-\kappa(\bar{T}-t)))
$$

Proof. From (4.1) we have for $s \in[t, \bar{T}]$

(6.3) $r_{s}=r_{t} \exp (-\kappa(s-t))+\bar{r}(1-\exp (-\kappa(s-t)))+\sigma \int_{t}^{s} \exp (-\kappa(s-u)) d Z_{u}$ 
K. FERGUSSON

Integrating both sides with respect to $s$ between $t$ and $\bar{T}$ gives

$$
\begin{aligned}
& \int_{t}^{\bar{T}} r_{s} d s \\
& =\int_{t}^{\bar{T}}\left(r_{t} \exp (-\kappa(s-t))+\bar{r}(1-\exp (-\kappa(s-t)))\right. \\
& \left.+\sigma \int_{t}^{s} \exp (-\kappa(s-u)) d Z_{u}\right) d s \\
& =\int_{t}^{\bar{T}} r_{t} \exp (-\kappa(s-t)) d s+\bar{r}(\bar{T}-t)+\bar{r} \frac{1}{\kappa}(\exp (-\kappa(\bar{T}-t))-1) \\
& +\sigma \int_{t}^{\bar{T}} \int_{t}^{s} \exp (-\kappa(s-u)) d Z_{u} d s \\
& =r_{t} \frac{1}{\kappa}(1-\exp (-\kappa(\bar{T}-t)))+\bar{r}\left(\bar{T}-t-\frac{1}{\kappa}(1-\exp (-\kappa(\bar{T}-t)))\right. \\
& +\sigma \frac{1}{\kappa} \int_{t}^{\bar{T}}(1-\exp (-\kappa(\bar{T}-u))) d Z_{u}
\end{aligned}
$$

which completes the proof.

A similar lemma applies to the Hull-White model.

Lemma 3. Let $r_{t}$ satisfy the Hull-White SDE (3.4). Then

$$
\int_{t}^{\bar{T}} r_{s} d s=r_{t} B(t, \bar{T})+\int_{t}^{\bar{T}} B(u, \bar{T})\{\theta(u)+a(u) b\} d u \int_{t}^{\bar{T}} B(u, \bar{T}) \sigma(u) d Z_{u}
$$

where

$$
B(t, \bar{T})=\int_{t}^{\bar{T}} \exp \left\{-\int_{t}^{s} a(\tau) d \tau\right\} d s
$$

The following proposition provides the formula for the savings account under the Vasicek short rate model.

Proposition 3. Let $r_{t}$ satisfy the Vasicek SDE (3.3). Then the SDE

$$
d B_{t}=r_{t} B_{t} d t
$$

of the savings account $B_{t}$ has the solution

$$
B_{\bar{T}}=B_{t} \exp \left(r_{t} B(t, \bar{T})+\bar{r}(\bar{T}-t-B(t, \bar{T}))+\sigma \int_{t}^{\bar{T}} B(u, \bar{T}) d Z_{u}\right)
$$

where $B(t, \bar{T})$ is as in $(6.2)$.

Proof. Combining (6.1) and (2.1) gives the formula for the savings account as

$$
\begin{aligned}
B_{\bar{T}} & =B_{t} \exp \left(\int_{t}^{\bar{T}} r_{s} d s\right) \\
& =B_{t} \exp \left(r_{t} B(t, \bar{T})+\bar{r}(\bar{T}-t-B(t, \bar{T}))+\sigma \int_{t}^{\bar{T}} B(u, \bar{T}) d Z_{u}\right),
\end{aligned}
$$

which completes the proof. 
ASYMPTOTICS OF BOND YIELDS AND VOLATILITIES FOR EXTENDED VASICEK MODEIS

From (6.8) we immediately see that the transition density function

$$
p_{B}\left(t, B_{t}, \bar{T}, B_{\bar{T}}\right)
$$

of the savings account value is a lognormal density function.

Proposition 4. Let $r_{t}$ satisfy the Vasicek SDE (3.3). Then the transition density function of the savings account value $B_{\bar{T}}$ is

$$
p_{B}\left(t, B_{t}, \bar{T}, B_{\bar{T}}\right)=\frac{1}{B_{\bar{T}} \sqrt{2 \pi v(t, \bar{T})}} \exp \left(-\frac{1}{2}\left(\log \left(B_{\bar{T}} / B_{t}\right)-m(t, \bar{T})\right)^{2} / v(t, \bar{T})\right),
$$

where

$$
\begin{aligned}
m(t, \bar{T}) & =r_{t} B(t, \bar{T})+\bar{r}(\bar{T}-t-B(t, \bar{T})) \\
v(t, \bar{T}) & =\frac{\sigma^{2}}{\kappa^{2}}\left(\bar{T}-t-B(t, \bar{T})-\frac{1}{2} \kappa B(t, \bar{T})^{2}\right) .
\end{aligned}
$$

Proof. From (6.8) we can write

$$
B_{\bar{T}}=B_{t} \exp (m(t, \bar{T})+\sqrt{v(t, \bar{T})} Z)
$$

where

$$
\begin{aligned}
m(t, \bar{T}) & =r_{t} B(t, \bar{T})+\bar{r}(\bar{T}-t-B(t, \bar{T})) \\
v(t, \bar{T}) & =\sigma^{2} \int_{t}^{\bar{T}} B(u, \bar{T})^{2} d u
\end{aligned}
$$

and $Z$ is a standard normal random variable. We can simplify the squared volatility $v(t, \bar{T})$ as follows

$$
\begin{aligned}
v(t, \bar{T}) & =\sigma^{2} \int_{t}^{\bar{T}} \frac{1}{\kappa^{2}}(1-\exp (-\kappa(\bar{T}-u)))^{2} d u \\
& =\frac{\sigma^{2}}{\kappa^{2}}\left((\bar{T}-t)-2 \frac{1-\exp (-\kappa(\bar{T}-t))}{\kappa}+\frac{1-\exp (-2 \kappa(\bar{T}-t))}{2 \kappa}\right) \\
& =\frac{\sigma^{2}}{\kappa^{2}}\left((\bar{T}-t)-2 B(t, \bar{T})+\frac{1-(1-\kappa B(t, \bar{T}))^{2}}{2 \kappa}\right) \\
& =\frac{\sigma^{2}}{\kappa^{2}}\left(\bar{T}-t-B(t, \bar{T})-\frac{1}{2} \kappa B(t, \bar{T})^{2}\right)
\end{aligned}
$$

and we have the result.

Therefore, we can write the conditional distribution of the savings account value as

$$
\log B_{\bar{T}} \sim N\left(\log B_{t}+m(t, \bar{T}), v(t, \bar{T})\right)
$$

given $B_{t}$ for $m(t, \bar{T})$ and $v(t, \bar{T})$ as in $(6.12)$, where $N(m, v)$ denotes the Gaussian distribution with mean $m$ and variance $v$.

Analogously, for the Hull-White model we can write the conditional distribution of the savings account value as

$$
\log B_{\bar{T}} \sim N\left(\log B_{t}+m(t, \bar{T}), v(t, \bar{T})\right)
$$


given $B_{t}$, where $m(t, \bar{T})$ and $v(t, \bar{T})$ are given by

$$
\begin{aligned}
m(t, \bar{T}) & =r_{t} \int_{t}^{\bar{T}} \exp \left\{-\int_{t}^{s} a(\tau) d \tau\right\} d s \\
& +\int_{t}^{\bar{T}}\left[\int_{t}^{s} \exp \left\{-\int_{u}^{s} a(\tau) d \tau\right\}(\theta(u)+a(u) b) d u\right] d s \\
v(t, \bar{T}) & =\int_{t}^{\bar{T}}\left[\int_{u}^{\bar{T}} \exp \left\{-\int_{u}^{s} a(\tau) d \tau\right\} d s\right]^{2} \sigma(u)^{2} d u .
\end{aligned}
$$

The transition density of the savings account allows us to calculate the zero coupon bond price in the following section.

\section{ZCB PRICE}

In the following lemma we calculate the $\bar{T}$-maturity zero coupon bond price $G_{\bar{T}}(t)$, given in $(2.6)$ and which can be rewritten as

$$
G_{\bar{T}}(t)=E\left(\frac{B_{t}}{B_{\bar{T}}} \mid \mathcal{A}_{t}\right)
$$

Lemma 4. Let $r_{t}$ satisfy the Vasicek $S D E$ (3.3). Then for time $t \in[0, \bar{T}]$ the $\bar{T}$-maturity $Z C B$ price is

$$
G_{\bar{T}}(t)=A(t, \bar{T}) \exp \left(-r_{t} B(t, \bar{T})\right)
$$

where

$$
B(t, \bar{T})=\frac{1-\exp (-\kappa(\bar{T}-t))}{\kappa}
$$

and

$$
A(t, \bar{T})=\exp \left(\left(\bar{r}-\frac{\sigma^{2}}{2 \kappa^{2}}\right)(B(t, \bar{T})-\bar{T}+t)-\frac{\sigma^{2}}{4 \kappa} B(t, \bar{T})^{2}\right)
$$

Proof. From (6.16)

$$
\log B_{\bar{T}} \sim N\left(\log B_{t}+m(t, \bar{T}), v(t, \bar{T})\right)
$$


ASYMPTOTICS OF BOND YIELDS AND VOLATILITIES FOR EXTENDED VASICEK MODEILS given $B_{t}$ and using (7.1) we have

$$
\begin{aligned}
G_{\bar{T}}(t) & =E\left(\frac{B_{t}}{B_{\bar{T}}} \mid \mathcal{A}_{t}\right) \\
& =B_{t} E\left(\exp \left(-\log B_{\bar{T}}\right) \mid \mathcal{A}_{t}\right) \\
& =B_{t} \exp \left(-E\left(\log B_{\bar{T}} \mid \mathcal{A}_{t}\right)+\frac{1}{2} V A R\left(B_{\bar{T}} \mid \mathcal{A}_{t}\right)\right) \\
& =B_{t} \exp \left(-\log B_{t}-m(t, \bar{T})+\frac{1}{2} v(t, \bar{T})\right) \\
& =\exp \left(-m(t, \bar{T})+\frac{1}{2} v(t, \bar{T})\right) \\
& =\exp \left(-r_{t} B(t, \bar{T})-\bar{r}(\bar{T}-t-B(t, \bar{T}))\right. \\
& \left.+\frac{\sigma^{2}}{2 \kappa^{2}}\left(\bar{T}-t-B(t, \bar{T})-\frac{1}{2} \kappa B(t, \bar{T})^{2}\right)\right) \\
& =\exp \left(-r_{t} B(t, \bar{T})\right) \exp \left(\left(-\bar{r}+\frac{\sigma^{2}}{2 \kappa^{2}}\right)(\bar{T}-t-B(t, \bar{T}))-\frac{\sigma^{2}}{4 \kappa} B(t, \bar{T})^{2}\right)
\end{aligned}
$$

which is the result.

A similar result can be proven for the Hull-White short rate model.

Lemma 5. Let $r_{t}$ satisfy the Hull-White $S D E$ (3.4). Then for time $t \in[0, \bar{T}]$ the $\bar{T}$-maturity $Z C B$ price is

$$
G_{\bar{T}}(t)=A(t, \bar{T}) \exp \left(-r_{t} B(t, \bar{T})\right)
$$

where

$$
B(t, \bar{T})=\int_{t}^{\bar{T}} \exp \left\{-\int_{t}^{s} a(\tau) d \tau\right\} d s
$$

and

$$
\begin{aligned}
A(t, \bar{T}) & =\exp \left(-\int_{t}^{\bar{T}}\left[\int_{t}^{s} \exp \left\{-\int_{u}^{s} a(\tau) d \tau\right\}(\theta(u)+a(u) b) d u\right] d s\right. \\
& \left.+\frac{1}{2} \int_{t}^{\bar{T}} B(u, \bar{T})^{2} \sigma(u)^{2} d u\right) .
\end{aligned}
$$

\section{Bond Yields and Forward Rates}

We investigate the asymptotic level of the yield curve under the Vasicek model. As a corollary of Lemma 4 we calculate the $\bar{T}$-maturity ZCB yield $h_{\bar{T}}(t)$, as given in

$$
h_{\bar{T}}(t)=-\frac{1}{\bar{T}-t} \log G_{\bar{T}}(t),
$$

as $\bar{T} \rightarrow \infty$, which we call the long ZCB yield.

Corollary 3. Let $r_{t}$ satisfy the Vasicek SDE (3.3). Then the long ZCB yield is

$$
h_{\infty}(t)=\bar{r}-\frac{\sigma^{2}}{2 \kappa^{2}} .
$$


FiguRE 5. Zero coupon yield curve under the Vasicek model based at 1871 .

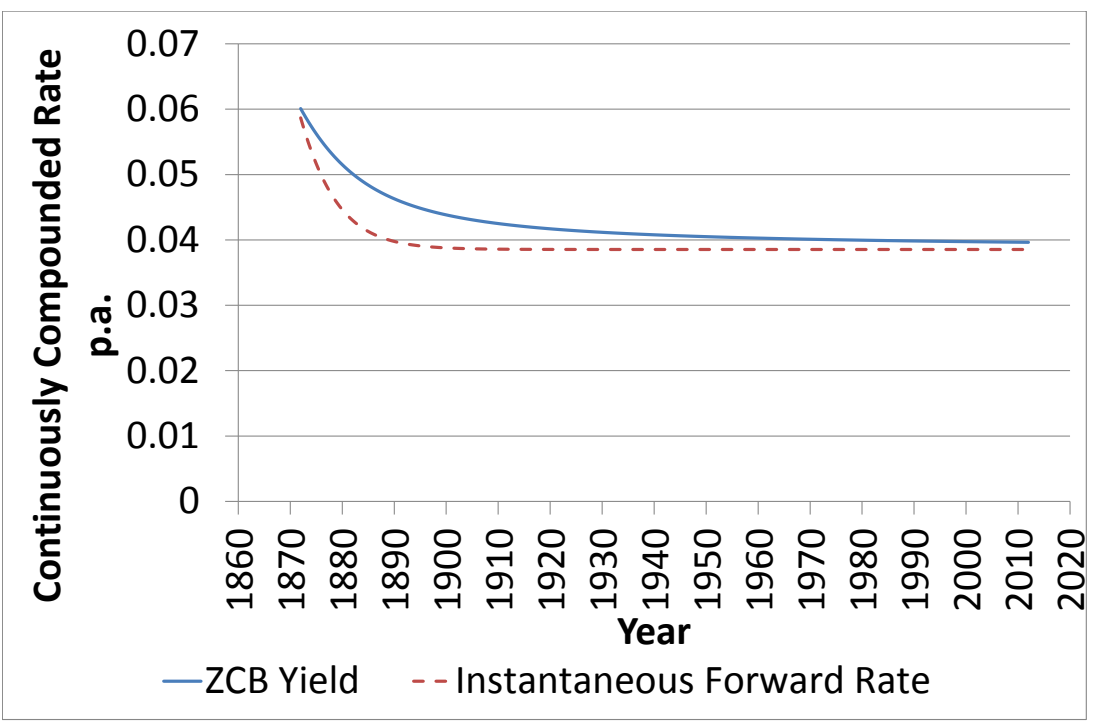

Proof. From (8.1), the ZCB yield is given by the formula

$$
\begin{aligned}
h_{\infty}(t) & =-\lim _{\bar{T} \rightarrow \infty} \frac{1}{\bar{T}-t} \log G_{\bar{T}}(t) \\
& =\lim _{\bar{T} \rightarrow \infty} \frac{1}{\bar{T}-t}\left(-\log A(t, \bar{T})+r_{t} B(t, \bar{T})\right) \\
& =\lim _{\bar{T} \rightarrow \infty} r_{t} \frac{B(t, \bar{T})}{\bar{T}-t}-\left(\bar{r}-\frac{\sigma^{2}}{2 \kappa^{2}}\right) \frac{B(t, \bar{T})-\bar{T}+t}{\bar{T}-t}+\frac{\sigma^{2}}{4 \kappa(\bar{T}-t)} B(t, \bar{T})^{2} .
\end{aligned}
$$

But $\lim _{\bar{T} \rightarrow \infty} B(t, \bar{T})=\frac{1}{\kappa}$ and, therefore, the long ZCB yield simplifies to $\bar{r}-\frac{\sigma^{2}}{2 \kappa^{2}}$.

In Figure 5 the continuously compounded yield curve is plotted as at the time of 1871 . We have an inverted yield curve and this portends an economic recession because decreasing forward rates indicate expectations of low inflation and low economic growth, as discussed in Harvey [1991].

For the Hull-White model, we have the following theorem which gives the long ZCB yield under suitable conditions on the functions $\theta(u), a(u)$ and $\sigma(u)$ in (3.4).

Theorem 1. Let $r_{t}$ satisfy the Hull-White SDE (3.4). Suppose further that as $u \rightarrow \infty$ we have

$$
\begin{gathered}
a(u) \rightarrow \bar{a}>0 \\
\theta(u) \rightarrow \bar{\theta} \in \mathbb{R} \\
\sigma(u) \rightarrow \bar{\sigma}>0 .
\end{gathered}
$$


Then the long $Z C B$ yield is

$$
h_{\infty}(t)=b+\frac{\bar{\theta}}{\bar{a}}-\frac{\bar{\sigma}^{2}}{2 \bar{a}^{2}} .
$$

Proof. We first show that the integral

$$
I=\int_{t}^{\bar{T}} \int_{t}^{s} \exp \left\{-\int_{u}^{s} a(\tau) d \tau\right\} \theta(u) d u d s
$$

is asymptotic to $\frac{\bar{\theta}}{\bar{a}}(\bar{T}-t)$ as $\bar{T} \rightarrow \infty$. We let $\epsilon$ be a fixed positive number less than one and choose the number $T_{\epsilon}>t$ such that for $u>T_{\epsilon},|a(u)-\bar{a}|<\epsilon$ and $|\theta(u)-\bar{\theta}|<\epsilon$. We also choose the number $\bar{T}_{\epsilon}$ such that

$$
\max \left\{\frac{1}{\bar{T}_{\epsilon}-t}, \frac{T_{\epsilon}-t}{\bar{T}_{\epsilon}-t}\right\}=\epsilon .
$$

We note that since $\epsilon<1$ we must have $\bar{T}_{\epsilon}>T_{\epsilon}$. Also, let the function $a(u)$ have lower and upper bounds $L_{a}$ and $U_{a}$ for $u \in[0, \infty)$. It is evident from the positivity of $a(u)$ that $0<L_{a} \leq U_{a}$. Finally, let $\theta(u)$ have lower and upper bounds $L_{\theta}$ and $U_{\theta}$ for $u \in[0, \infty)$.

Now, for $\bar{T}>\bar{T}_{\epsilon}$ we show that the integral $I$ in (8.6) is close to $\frac{\bar{\theta}}{\bar{a}}(\bar{T}-t)$. Without loss of generality, we assume that $\theta(u) \geq 0$. Indeed, if $L_{\theta}<0$ we can express the integral $I$ as a sum of two integrals, that is,

$I=\int_{t}^{\bar{T}} \int_{t}^{s} \exp \left\{-\int_{u}^{s} a(\tau) d \tau\right\}\left(\theta(u)-L_{\theta}\right) d u d s+L_{\theta} \int_{t}^{\bar{T}} \int_{t}^{s} \exp \left\{-\int_{u}^{s} a(\tau) d \tau\right\} d u d s$,

and apply the following reasoning to each of the integrals, obtaining the desired result.

Partitioning the region of integration into three subregions, we write

$$
I=\left\{\int_{t}^{T_{\epsilon}} \int_{t}^{s}+\int_{T_{\epsilon}}^{\bar{T}} \int_{T_{\epsilon}}^{s}+\int_{T_{\epsilon}}^{\bar{T}} \int_{t}^{T_{\epsilon}}\right\} \exp \left\{-\int_{u}^{s} a(\tau) d \tau\right\} \theta(u) d u d s=I_{A}+I_{B}+I_{C} .
$$

Using the lower and upper bounds of $a(u)$ and $\theta(u)$ on $[0, \infty)$ we have

$$
\int_{t}^{T_{\epsilon}} \int_{t}^{s} \exp \left\{-(s-u) U_{a}\right\} L_{\theta} d u d s<I_{A}<\int_{t}^{T_{\epsilon}} \int_{t}^{s} \exp \left\{-(s-u) L_{a}\right\} U_{\theta} d u d s,
$$

which gives the inequalities

$$
\begin{aligned}
& \frac{L_{\theta}}{U_{a}}\left(T_{\epsilon}-t\right)-\frac{L_{\theta}}{U_{a}^{2}}<\frac{L_{\theta}}{U_{a}}\left(T_{\epsilon}-t\right)-\frac{L_{\theta}}{U_{a}^{2}}\left(1-\exp \left\{-U_{a}\left(T_{\epsilon}-t\right)\right\}\right)<I_{A} \\
& I_{A}<\frac{U_{\theta}}{L_{a}}\left(T_{\epsilon}-t\right)-\frac{U_{\theta}}{L_{a}^{2}}\left(1-\exp \left\{-L_{a}\left(T_{\epsilon}-t\right)\right\}\right)<\frac{U_{\theta}}{L_{a}}\left(T_{\epsilon}-t\right) .
\end{aligned}
$$

Similarly, using the lower and upper bounds of $a(u)$ and $\theta(u)$ on $\left[T_{\epsilon}, \infty\right)$, that is,

$$
\begin{gathered}
\bar{a}-\epsilon<a(u)<\bar{a}+\epsilon \\
\bar{\theta}-\epsilon<\theta(u)<\bar{\theta}+\epsilon,
\end{gathered}
$$

we have the inequalities

$$
\frac{\bar{\theta}-\epsilon}{\bar{a}+\epsilon}\left(\bar{T}-T_{\epsilon}\right)-\frac{\bar{\theta}-\epsilon}{(\bar{a}+\epsilon)^{2}}<I_{B}<\frac{\theta+\epsilon}{\bar{a}-\epsilon}\left(\bar{T}-T_{\epsilon}\right) .
$$


K. FERGUSSON

Finally, we have the inequalities for $I_{C}$

$$
0 \leq I_{C}<\int_{T_{\epsilon}}^{\bar{T}} \int_{t}^{T_{\epsilon}} \exp \left\{-L_{a}(s-u)\right\} U_{\theta} d u d s<\frac{U_{\theta}}{L_{a}^{2}} .
$$

Combining Inequalities (8.11), (8.13) and (8.14) gives, for $\bar{T}>\bar{T}_{\epsilon}$,

$$
\begin{aligned}
& \bar{a} \frac{L_{\theta}}{U_{a}} \frac{T_{\epsilon}-t}{\bar{T}-t}-\frac{\bar{a}}{\bar{T}-t} \frac{L_{\theta}}{U_{a}^{2}}+\frac{\bar{\theta}-\epsilon}{1-\epsilon / \bar{a}} \frac{\bar{T}-T_{\epsilon}}{\bar{T}-t}-\frac{\bar{a}}{\bar{T}-t} \frac{\bar{\theta}-\epsilon}{(\bar{a}+\epsilon)^{2}} \\
& <\frac{\bar{a}}{\bar{T}-t}\left(I_{A}+I_{B}+I_{C}\right)=\frac{\bar{a}}{\bar{T}-t} I \\
& <\bar{a} \frac{U_{\theta}}{L_{a}} \frac{T_{\epsilon}-t}{\bar{T}-t}+\frac{\bar{\theta}+\epsilon}{1-\epsilon / \bar{a}} \frac{\bar{T}-T_{\epsilon}}{\bar{T}-t}+\frac{\bar{a}}{\bar{T}-t} \frac{U_{\theta}}{L_{a}^{2}} .
\end{aligned}
$$

Making use of our choice of $\bar{T}_{\epsilon}$ in (8.7) the above inequalities simplify to

$$
-\bar{a} \frac{L_{\theta}}{U_{a}^{2}} \epsilon+\frac{\bar{\theta}-\epsilon}{1-\epsilon / \bar{a}}(1-\epsilon)-\bar{a} \epsilon \frac{\bar{\theta}-\epsilon}{(\bar{a}+\epsilon)^{2}}<\frac{\bar{a}}{\bar{T}-t} I<\bar{a} \frac{U_{\theta}}{L_{a}} \epsilon+\frac{\bar{\theta}+\epsilon}{1-\epsilon / \bar{a}}+\bar{a} \epsilon \frac{U_{\theta}}{L_{a}^{2}},
$$

for $\bar{T}>\bar{T}_{\epsilon}$. Thus, (8.16) demonstrates that $\frac{\bar{a}}{\bar{T}-t} I$ can be made arbitrarily close to $\bar{\theta}$, as $\bar{T} \rightarrow \infty$. The proofs of the limits

$$
\begin{array}{r}
\lim _{\bar{T} \rightarrow \infty} \frac{1}{\bar{T}-t} \int_{t}^{\bar{T}} \int_{t}^{s} \exp \left\{-\int_{u}^{s} a(\tau) d \tau\right\} a(u) b d u d s=b \\
\lim _{\bar{T} \rightarrow \infty} \frac{1}{\bar{T}-t} \int_{t}^{\bar{T}}\left[\int_{u}^{\bar{T}} \exp \left\{-\int_{u}^{s} a(\tau) d \tau\right\} d s\right]^{2} \sigma(u)^{2} d u=\frac{\bar{\sigma}^{2}}{\bar{a}}
\end{array}
$$

are essentially the same. Therefore, as $\bar{T} \rightarrow \infty$, the long ZCB yield simplifies to $b+\frac{\bar{\theta}}{\bar{a}}-\frac{\bar{\sigma}^{2}}{2 \bar{a}^{2}}$.

We calculate the forward rate $g_{\bar{T}}(t)$, given by

$$
g_{\bar{T}}(t)=-\frac{\partial}{\partial \bar{T}} \log G_{\bar{T}}(t)
$$

Lemma 6. For time $t \in[0, \bar{T}]$ the forward rate is computed to be

$$
g_{\bar{T}}(t)=\left(r_{t}-\bar{r}\right) \exp (-\kappa(\bar{T}-t))+\bar{r}-\frac{\sigma^{2}}{2 \kappa^{2}}(1-\exp (-\kappa(\bar{T}-t)))^{2} .
$$

Proof. Using (8.18) and (7.2) we have

$$
\begin{aligned}
g_{\bar{T}}(t) & =-\frac{\partial}{\partial \bar{T}} \log G_{\bar{T}}(t) \\
& =-\frac{\partial}{\partial \bar{T}}\left\{-\left(r_{t}-\bar{r}\right) \frac{(1-\exp (-\kappa(\bar{T}-t)))}{\kappa}-\bar{r}(\bar{T}-t)\right. \\
& \left.+\frac{\sigma^{2}}{2 \kappa^{2}}\left((\bar{T}-t)-2 \frac{1-\exp (-\kappa(\bar{T}-t))}{\kappa}+\frac{1-\exp (-2 \kappa(\bar{T}-t))}{2 \kappa}\right)\right\} \\
& =\left(r_{t}-\bar{r}\right) \exp (-\kappa(\bar{T}-t))+\bar{r} \\
& -\frac{\sigma^{2}}{2 \kappa^{2}}(1-2 \exp (-\kappa(\bar{T}-t))+\exp (-2 \kappa(\bar{T}-t)))
\end{aligned}
$$

and simplifying gives the result. 
As a corollary of this lemma we calculate directly the asymptotic instantaneous forward rate.

Corollary 4. For the Vasicek short rate model, the asymptotic instantaneous forward rate is

$$
g_{\infty}(t)=\bar{r}-\frac{\sigma^{2}}{2 \kappa^{2}}
$$

In Figure 5 the instantaneous forward rate $g_{\bar{T}}$ is plotted and can be seen to be asymptotic to $g_{\infty}(t)=0.0385$ based upon the parameters in (5.4).

\section{Expectations InVolving $G_{\bar{T}}(t)$}

Motivated by our goal of pricing call and put options on zero coupon bonds, we seek formulae for the following expectations

$$
\begin{aligned}
& f_{1}(t, T, K, \bar{T})=E\left(\exp \left(-\int_{t}^{T} r_{s} d s\right) G_{\bar{T}}(T) \mathbf{1}_{G_{\bar{T}}(T)>K} \mid \mathcal{A}_{t}\right) \\
& f_{2}(t, T, K, \bar{T})=E\left(\exp \left(-\int_{t}^{T} r_{s} d s\right) G_{\bar{T}}(T) \mathbf{1}_{G_{\bar{T}}(T) \leq K} \mid \mathcal{A}_{t}\right) \\
& f_{3}(t, T, K, \bar{T})=E\left(\exp \left(-\int_{t}^{T} r_{s} d s\right) \mathbf{1}_{G_{\bar{T}}(T)>K} \mid \mathcal{A}_{t}\right) \\
& f_{4}(t, T, K, \bar{T})=E\left(\exp \left(-\int_{t}^{T} r_{s} d s\right) \mathbf{1}_{G_{\bar{T}}(T) \leq K} \mid \mathcal{A}_{t}\right) \\
& f_{5}(t, T, K, \bar{T})=E\left(\exp \left(-\int_{t}^{T} r_{s} d s\right)\left(G_{\bar{T}}(T)-K\right)^{+} \mid \mathcal{A}_{t}\right) \\
& f_{6}(t, T, K, \bar{T})=E\left(\exp \left(-\int_{t}^{T} r_{s} d s\right)\left(K-G_{\bar{T}}(T)\right)^{+} \mid \mathcal{A}_{t}\right)
\end{aligned}
$$

where $0 \leq t<T<\bar{T}$ and $K>0$. These expectations correspond to prices of various call and put options on zero coupon bonds under the Vasicek short rate model.

It is well known that the Vasicek short rate model is an example of a Gaussian interest rate model and that for such models the prices of call options on zero coupon bonds employ the Black-Scholes option pricing formula. We establish that the Black-Scholes formula applies when performing actuarial pricing of contingent claims, employing the following four lemmas which culminate in three subsequent theorems.

Lemma 7. We have

$$
\begin{aligned}
E(\exp (\alpha Z)) & =\exp \left(\frac{1}{2} \alpha^{2}\right) \\
E\left(\exp (\alpha Z) 1_{Z>z}\right) & =\exp \left(\frac{1}{2} \alpha^{2}\right)(1-N(z-\alpha)) \\
E\left(\exp (\alpha Z) 1_{Z \leq z}\right) & =\exp \left(\frac{1}{2} \alpha^{2}\right) N(z-\alpha)
\end{aligned}
$$


Proof. We have

$$
\begin{aligned}
E(\exp (\alpha Z)) & =\int_{-\infty}^{\infty} \exp (\alpha u) n(u) d u \\
& =\int_{-\infty}^{\infty} \exp \left(\frac{1}{2} \alpha^{2}\right) n(u-\alpha) d u \\
& =\exp \left(\frac{1}{2} \alpha^{2}\right) \int_{-\infty}^{\infty} n(v) d v \\
& =\exp \left(\frac{1}{2} \alpha^{2}\right)
\end{aligned}
$$

Next we have

$$
\begin{aligned}
E\left(\exp (\alpha Z) \mathbf{1}_{Z>z}\right) & =\int_{z}^{\infty} \exp (\alpha u) n(u) d u \\
& =\int_{z}^{\infty} \exp \left(\frac{1}{2} \alpha^{2}\right) n(u-\alpha) d u \\
& =\exp \left(\frac{1}{2} \alpha^{2}\right) \int_{z-\alpha}^{\infty} n(v) d v \\
& =\exp \left(\frac{1}{2} \alpha^{2}\right) E\left(\mathbf{1}_{Z>z-\alpha}\right) \\
& =\exp \left(\frac{1}{2} \alpha^{2}\right)(1-N(z-\alpha)),
\end{aligned}
$$

which is the second result. The third result is obtained by transposing the identity

$$
E\left(\exp (\alpha Z) \mathbf{1}_{Z>z}\right)+E\left(\exp (\alpha Z) \mathbf{1}_{Z \leq z}\right)=E(\exp (\alpha Z))
$$

and applying the first two results.

Lemma 8. Let $Y$ be a normally distributed random variable. Then for any real number $y$ we have

$$
E\left(\exp (Y) \mathbf{1}_{Y \leq y}\right)=E(\exp (Y)) \times E\left(\mathbf{1}_{Y \leq y-V A R(Y)}\right)
$$

and

$$
E\left(\exp (Y) \mathbf{1}_{Y>y}\right)=E(\exp (Y)) \times E\left(\boldsymbol{1}_{Y>y-V A R(Y)}\right) .
$$

Proof. Let us write the random variable $Y$ in the form $Y=\mu+\sigma Z$, where $Z$ is a standard normal random variable and $\sigma$ is a positive real number. Clearly, $E(Y)=\mu$ and $V A R(Y)=\sigma^{2}$. Also $E(\exp (Y))=\exp \left(\mu+\frac{1}{2} \sigma^{2}\right)$. Then

$$
\begin{aligned}
E\left(\exp (Y) \mathbf{1}_{Y \leq y}\right) & =E\left(\exp (\mu+\sigma Z) \mathbf{1}_{\mu+\sigma Z \leq y}\right) \\
& =\exp (\mu) E\left(\exp (\sigma Z) \mathbf{1}_{Z \leq(y-\mu) / \sigma}\right)
\end{aligned}
$$

and from Lemma 7 we have

$$
\begin{aligned}
E\left(\exp (Y) \mathbf{1}_{Y \leq y}\right) & =\exp (\mu) \exp \left(\frac{1}{2} \sigma^{2}\right) E\left(\mathbf{1}_{Z \leq(y-\mu) / \sigma-\sigma}\right) \\
& =E(\exp (Y)) \times E\left(\mathbf{1}_{Z \leq(y-\mu) / \sigma-\sigma}\right) \\
& =E(\exp (Y)) \times E\left(\mathbf{1}_{Y \leq y-\sigma^{2}}\right)
\end{aligned}
$$

as required. Also the second equality emerges after applying the relation

$$
E\left(\exp (Y) \mathbf{1}_{Y>y}\right)=E\left(\exp (Y)\left(1-\mathbf{1}_{Y \leq y}\right)\right)=E(\exp (Y))-E\left(\exp (Y) \mathbf{1}_{Y \leq y}\right)
$$

to the first equality. 
In the following lemma we state an extension of the above lemma.

Lemma 9. Let $Y_{1}$ and $Y_{2}$ be normally distributed random variables. Then for any real number $y$,

$$
E\left(\exp \left(Y_{1}\right) \boldsymbol{1}_{Y_{2} \leq y}\right)=E\left(\exp \left(Y_{1}\right)\right) \times E\left(\boldsymbol{1}_{Y_{2} \leq y-C O V\left(Y_{1}, Y_{2}\right)}\right)
$$

Also we have

$$
E\left(\exp \left(Y_{1}\right) \boldsymbol{1}_{Y_{2}>y}\right)=E\left(\exp \left(Y_{1}\right)\right) \times E\left(\boldsymbol{1}_{Y_{2}>y-\operatorname{COV}\left(Y_{1}, Y_{2}\right)}\right)
$$

Proof. We let

$$
Y_{2}^{\prime}=Y_{2}-\beta Y_{1}
$$

where $\beta=\operatorname{COV}\left(Y_{1}, Y_{2}\right) / \operatorname{VAR}\left(Y_{1}\right)$. This allows us to write $Y_{2}$ as a linear combination of two uncorrelated random variables $Y_{1}$ and $Y_{2}^{\prime}$ as follows:

$$
Y_{2}=\beta Y_{1}+Y_{2}^{\prime}
$$

If $\beta=0$, then $Y_{1}$ and $Y_{2}$ are uncorrelated and, because both are normally distributed random variables, are therefore independent which gives, by Lemma 8, the result. Henceforth we assume $\beta \neq 0$ and we have, by Lemma 8 ,

$$
\begin{aligned}
E\left(\exp \left(Y_{1}\right) \mathbf{1}_{Y_{2} \leq y}\right) & =E\left(\exp \left(Y_{1}\right) \mathbf{1}_{\beta Y_{1}+Y_{2}^{\prime} \leq y}\right) \\
& =E\left(E\left(\exp \left(Y_{1}\right) \mathbf{1}_{\beta Y_{1}+Y_{2}^{\prime} \leq y} \mid Y_{2}^{\prime}\right)\right) \\
& =E\left(E\left(\exp \left(Y_{1}\right) \mathbf{1}_{Y_{1} \leq \frac{1}{\beta}\left(y-Y_{2}^{\prime}\right)} \mid Y_{2}^{\prime}\right)\right) \text { for } \beta>0 .
\end{aligned}
$$

We remark that for $\beta<0$ the inequality is reversed in the indicator function above, yet an identical result to that which follows is obtained. We apply Lemma 8 to evaluate the inner expectation, giving

$$
\begin{aligned}
& E\left(E\left(\exp \left(Y_{1}\right) \mathbf{1}_{Y_{1} \leq \frac{1}{\beta}\left(y-Y_{2}^{\prime}\right)} \mid Y_{2}^{\prime}\right)\right) \\
& =E\left(E\left(\exp \left(Y_{1}\right)\right) E\left(\mathbf{1}_{Y_{1} \leq \frac{1}{\beta}\left(y-Y_{2}^{\prime}\right)-V A R\left(Y_{1}\right)} \mid Y_{2}^{\prime}\right)\right) \\
& =E\left(\exp \left(Y_{1}\right)\right) E\left(E\left(\mathbf{1}_{\beta Y_{1}+Y_{2}^{\prime} \leq y-\beta V A R\left(Y_{1}\right)} \mid Y_{2}^{\prime}\right)\right) \\
& =E\left(\exp \left(Y_{1}\right)\right) E\left(\mathbf{1}_{Y_{2} \leq y-\beta V A R\left(Y_{1}\right)}\right) \\
& =E\left(\exp \left(Y_{1}\right)\right) E\left(\mathbf{1}_{Y_{2} \leq y-\operatorname{COV}\left(Y_{1}, Y_{2}\right)}\right)
\end{aligned}
$$

which is the first equality. The second equality also follows similarly.

We can readily prove the formulae for ZCB call and put options using Lemma 9 when the integral of the short rate is a normally distributed random variable whose variance parameter is a deterministic function, that is when the following condition holds:

Condition 1. The integral $\int_{t}^{T} r_{s} d s$ is normally distributed, that is,

$$
\int_{t}^{T} r_{s} d s \sim N(m, v),
$$

where the parameter $v$ is a deterministic function involving the parameters $\bar{r}, \kappa, \sigma$, $t$ and $T$.

This condition is satisfied by the Ho-Lee short rate model, the Hull-White short rate model and various extended versions of these. Therefore, our lemmas apply to these models, which result in a proof of the Black-Scholes formula for options on zero coupon bonds under each of these models. 
Lemma 10. Let $r_{t}$ be a process for the short rate which satisfies Condition 1 and let

$$
G_{\bar{T}}(T)=E\left(\exp \left(-\int_{T}^{\bar{T}} r_{s} d s\right) \mid \mathcal{A}_{T}\right) .
$$

Then the random variable $L$ conditional on information up to time $t$, given by

$$
L=\log G_{\bar{T}}(T)
$$

is normally distributed whose expected value satisfies

$$
\begin{aligned}
E\left(L \mid \mathcal{A}_{t}\right) & =\log G_{\bar{T}}(t) / G_{T}(t) \\
& -\frac{1}{2} V A R\left(L \mid \mathcal{A}_{t}\right)+\operatorname{COV}\left(L, \int_{t}^{T} r_{s} d s \mid \mathcal{A}_{t}\right)
\end{aligned}
$$

and whose variance $\operatorname{VAR}\left(L \mid \mathcal{A}_{t}\right)$ satisfies

$$
\operatorname{VAR}\left(L \mid \mathcal{A}_{t}\right)=\operatorname{VAR}\left(E\left(\int_{T}^{\bar{T}} r_{s} d s \mid \mathcal{A}_{T}\right) \mid \mathcal{A}_{t}\right) .
$$

Proof. Because $\int_{0}^{t} r_{s} d s$ is normally distributed we have

$$
\begin{aligned}
G_{\bar{T}}(T) & =E\left(\exp \left(-\int_{T}^{\bar{T}} r_{s} d s\right) \mid \mathcal{A}_{T}\right) \\
& =E\left(\exp \left(-\int_{t}^{\bar{T}} r_{s} d s+\int_{t}^{T} r_{s} d s\right) \mid \mathcal{A}_{T}\right) \\
& =\exp \left(E\left(-\int_{t}^{\bar{T}} r_{s} d s+\int_{t}^{T} r_{s} d s \mid \mathcal{A}_{T}\right)\right. \\
& \left.+\frac{1}{2} V A R\left(\int_{t}^{\bar{T}} r_{s} d s \mid \mathcal{A}_{T}\right)\right),
\end{aligned}
$$

where we have used Condition 1, namely that $\operatorname{VAR}\left(\int_{t}^{T} r_{s} d s \mid \mathcal{A}_{T}\right)=0$, and the properties of the lognormal distribution. Therefore, the conditional random variable $L$ given the information available at time $t$ is given by

$$
L=\log G_{\bar{T}}(T)=-E\left(\int_{t}^{\bar{T}} r_{s} d s \mid \mathcal{A}_{T}\right)+\int_{t}^{T} r_{s} d s+\frac{1}{2} V A R\left(\int_{t}^{\bar{T}} r_{s} d s \mid \mathcal{A}_{T}\right)
$$

and is normally distributed.

Its expected value is

$$
\begin{aligned}
E\left(L \mid \mathcal{A}_{t}\right) & =-E\left(\int_{t}^{\bar{T}} r_{s} d s \mid \mathcal{A}_{t}\right)+E\left(\int_{t}^{T} r_{s} d s \mid \mathcal{A}_{t}\right) \\
& +\frac{1}{2} E\left(\operatorname{VAR}\left(\int_{t}^{\bar{T}} r_{s} d s \mid \mathcal{A}_{T}\right) \mid \mathcal{A}_{t}\right)
\end{aligned}
$$


ASYMPTOTICS OF BOND YIELDS AND VOLATILITIES FOR EXTENDED VASICEK MODELS

which simplifies to

$$
\begin{aligned}
E\left(L \mid \mathcal{A}_{t}\right) & =-E\left(\int_{t}^{\bar{T}} r_{s} d s \mid \mathcal{A}_{t}\right)+E\left(\int_{t}^{T} r_{s} d s \mid \mathcal{A}_{t}\right) \\
& +\frac{1}{2} V A R\left(\int_{t}^{\bar{T}} r_{s} d s \mid \mathcal{A}_{T}\right)
\end{aligned}
$$

because the variance $\operatorname{VAR}\left(\int_{t}^{\bar{T}} r_{s} d s \mid \mathcal{A}_{T}\right)$ is deterministic, as demonstrated by

$$
\begin{aligned}
\operatorname{VAR}\left(\int_{t}^{\bar{T}} r_{s} d s \mid \mathcal{A}_{T}\right) & =V A R\left(\int_{t}^{T} r_{s} d s+\int_{T}^{\bar{T}} r_{s} d s \mid \mathcal{A}_{T}\right) \\
& =V A R\left(\int_{T}^{\bar{T}} r_{s} d s \mid \mathcal{A}_{T}\right) \\
& =v(T, \bar{T}) .
\end{aligned}
$$

To simplify (9.25) we note that

$$
\begin{aligned}
& \log G_{\bar{T}}(t)=-E\left(\int_{t}^{\bar{T}} r_{s} d s \mid \mathcal{A}_{t}\right)+\frac{1}{2} V A R\left(\int_{t}^{\bar{T}} r_{s} d s \mid \mathcal{A}_{t}\right) \\
& \log G_{T}(t)=-E\left(\int_{t}^{T} r_{s} d s \mid \mathcal{A}_{t}\right)+\frac{1}{2} V A R\left(\int_{t}^{T} r_{s} d s \mid \mathcal{A}_{t}\right)
\end{aligned}
$$

and that, by virtue of the Law of Total Variance,

$$
V A R(X)=V A R(E(X \mid Y))+E(V A R(X \mid Y)),
$$

we have

$$
\operatorname{VAR}\left(\int_{t}^{\bar{T}} r_{s} d s \mid \mathcal{A}_{t}\right)=\operatorname{VAR}\left(E\left(\int_{t}^{\bar{T}} r_{s} d s \mid \mathcal{A}_{T}\right) \mid \mathcal{A}_{t}\right)+\operatorname{VAR}\left(\int_{t}^{\bar{T}} r_{s} d s \mid \mathcal{A}_{T}\right) .
$$

Therefore, we can rewrite (9.25) as

$$
\begin{aligned}
E\left(L \mid \mathcal{A}_{t}\right) & =\log G_{\bar{T}}(t)-\frac{1}{2} V A R\left(\int_{t}^{\bar{T}} r_{s} d s \mid \mathcal{A}_{t}\right) \\
& -\log G_{T}(t)+\frac{1}{2} V A R\left(\int_{t}^{T} r_{s} d s \mid \mathcal{A}_{t}\right) \\
& +\frac{1}{2} V A R\left(\int_{t}^{\bar{T}} r_{s} d s \mid \mathcal{A}_{T}\right) \\
& =\log G_{\bar{T}}(t) / G_{T}(t) \\
& -\frac{1}{2} V A R\left(E\left(\int_{t}^{\bar{T}} r_{s} d s \mid \mathcal{A}_{T}\right) \mid \mathcal{A}_{t}\right)-\frac{1}{2} V A R\left(\int_{t}^{\bar{T}} r_{s} d s \mid \mathcal{A}_{T}\right) \\
& +\frac{1}{2} V A R\left(\int_{t}^{T} r_{s} d s \mid \mathcal{A}_{t}\right)+\frac{1}{2} V A R\left(\int_{t}^{\bar{T}} r_{s} d s \mid \mathcal{A}_{T}\right) \\
& =\log G_{\bar{T}}(t) / G_{T}(t) \\
& -\frac{1}{2} V A R\left(E\left(\int_{t}^{\bar{T}} r_{s} d s \mid \mathcal{A}_{T}\right) \mid \mathcal{A}_{t}\right)+\frac{1}{2} V A R\left(\int_{t}^{T} r_{s} d s \mid \mathcal{A}_{t}\right) .
\end{aligned}
$$


Transposing (9.23) gives

$$
E\left(\int_{t}^{\bar{T}} r_{s} d s \mid \mathcal{A}_{T}\right)=-L+\int_{t}^{T} r_{s} d s+\frac{1}{2} V A R\left(\int_{t}^{\bar{T}} r_{s} d s \mid \mathcal{A}_{T}\right)
$$

and taking the variance of both sides gives

$$
\begin{aligned}
& \operatorname{VAR}\left(E\left(\int_{t}^{\bar{T}} r_{s} d s \mid \mathcal{A}_{T}\right) \mid \mathcal{A}_{t}\right)=\operatorname{VAR}\left(L \mid \mathcal{A}_{t}\right)+\operatorname{VAR}\left(\int_{t}^{T} r_{s} d s \mid \mathcal{A}_{t}\right) \\
& \quad-2 \operatorname{COV}\left(L, \int_{t}^{T} r_{s} d s \mid \mathcal{A}_{t}\right) .
\end{aligned}
$$

Substituting this variance formula into (9.30) gives (9.20). The formula for the variance $(9.21)$ is easily deduced by rewriting $(9.23)$ as

$$
L=-E\left(\int_{T}^{\bar{T}} r_{s} d s \mid \mathcal{A}_{T}\right)+\frac{1}{2} V A R\left(\int_{t}^{\bar{T}} r_{s} d s \mid \mathcal{A}_{T}\right)
$$

and taking variances of both sides.

Theorem 2. Let $r_{t}$ be a process for the short rate which satisfies Condition 1. Then the formulae for the expectations $f_{1}$ and $f_{2}$ in (9.1) are given by

$$
\begin{aligned}
& f_{1}(t, T, K, \bar{T})=G_{\bar{T}}(t) N\left(d_{1}\right) \\
& f_{2}(t, T, K, \bar{T})=G_{\bar{T}}(t)\left(1-N\left(d_{1}\right)\right),
\end{aligned}
$$

where

$$
\begin{aligned}
d_{1} & =\frac{1}{2} \sigma_{G}+\frac{1}{\sigma_{G}} \log \frac{G_{\bar{T}}(t)}{G_{T}(t) K} \\
\sigma_{G}^{2} & =V A R\left(E\left(\int_{T}^{\bar{T}} r_{s} d s \mid \mathcal{A}_{T}\right) \mid \mathcal{A}_{t}\right) .
\end{aligned}
$$

Proof. The price of the asset-or-nothing call option on $G_{\bar{T}}$ is

$$
\begin{aligned}
f_{1}(t, T, K, \bar{T}) & =E\left(\exp \left\{-\int_{t}^{T} r_{s} d s\right\} G_{\bar{T}}(T) \mathbf{1}_{G_{\bar{T}}(T)>K} \mid \mathcal{A}_{t}\right) \\
& =E\left(\exp \left\{-\int_{t}^{T} r_{s} d s\right\} E\left(\exp \left\{-\int_{T}^{\bar{T}} r_{s} d s\right\} \mid \mathcal{A}_{T}\right) \mathbf{1}_{L>\log K} \mid \mathcal{A}_{t}\right) \\
& =E\left(\exp \left\{-\int_{t}^{\bar{T}} r_{s} d s\right\} \mathbf{1}_{L>\log K} \mid \mathcal{A}_{t}\right) .
\end{aligned}
$$

We can apply Lemma 9 to the right hand side of (9.36) to give

$$
\begin{aligned}
& f_{1}(t, T, K, \bar{T}) \\
& =E\left(\exp \left\{-\int_{t}^{\bar{T}} r_{s} d s\right\} \mid \mathcal{A}_{t}\right) \times E\left(\mathbf{1}_{L>\log K-\operatorname{COV}\left(-\int_{t}^{\bar{T}} r_{s} d s, L \mid \mathcal{A}_{t}\right)} \mid \mathcal{A}_{t}\right) \\
& =G_{\bar{T}}(t) E\left(\mathbf{1}_{Z>z_{1}}\right)
\end{aligned}
$$

for a standard normal random variable $Z$ where

$$
z_{1}=\frac{1}{\sqrt{V A R(L)}}\left(\log K+\operatorname{COV}\left(\int_{t}^{\bar{T}} r_{s} d s, L \mid \mathcal{A}_{t}\right)-E(L)\right) .
$$


ASYMPTOTICS OF BOND YIELDS AND VOLATILITIES FOR EXTENDED VASICEK MODELS

The expression $\log K+C O V\left(\int_{t}^{\bar{T}} r_{s} d s, L \mid \mathcal{A}_{t}\right)-E(L)$ can be simplified using (9.30) to give

$$
\begin{aligned}
& \log K G_{T}(t) / G_{\bar{T}}(t)+\operatorname{COV}\left(\int_{t}^{\bar{T}} r_{s} d s, L \mid \mathcal{A}_{t}\right) \\
& +\frac{1}{2} V A R\left(L \mid \mathcal{A}_{t}\right)-\operatorname{COV}\left(L, \int_{t}^{T} r_{s} d s \mid \mathcal{A}_{t}\right) \\
& =\log K G_{T}(t) / G_{\bar{T}}(t)+\operatorname{COV}\left(\int_{T}^{\bar{T}} r_{s} d s, L \mid \mathcal{A}_{t}\right) \\
& +\frac{1}{2} V A R\left(L \mid \mathcal{A}_{t}\right) .
\end{aligned}
$$

From (9.33) we have

$$
\begin{aligned}
& \operatorname{COV}\left(\int_{T}^{\bar{T}} r_{s} d s, L \mid \mathcal{A}_{t}\right) \\
& =-\operatorname{COV}\left(\int_{T}^{\bar{T}} r_{s} d s, E\left(\int_{T}^{\bar{T}} r_{s} d s \mid \mathcal{A}_{T}\right) \mid \mathcal{A}_{t}\right) \\
& =-E\left(\int_{T}^{\bar{T}} r_{s} d s \times E\left(\int_{T}^{\bar{T}} r_{s} d s \mid \mathcal{A}_{T}\right) \mid \mathcal{A}_{t}\right) \\
& +E\left(\int_{T}^{\bar{T}} r_{s} d s \mid \mathcal{A}_{t}\right) \times E\left(E\left(\int_{T}^{\bar{T}} r_{s} d s \mid \mathcal{A}_{T}\right) \mid \mathcal{A}_{t}\right) .
\end{aligned}
$$

Using the law of total covariance, we have

$$
\begin{aligned}
& \operatorname{COV}\left(\int_{T}^{\bar{T}} r_{s} d s, L \mid \mathcal{A}_{t}\right) \\
& =-E\left(E\left(\int_{T}^{\bar{T}} r_{s} d s \times E\left(\int_{T}^{\bar{T}} r_{s} d s \mid \mathcal{A}_{T}\right) \mid \mathcal{A}_{T}\right) \mid \mathcal{A}_{t}\right)+\left\{E\left(\int_{T}^{\bar{T}} r_{s} d s \mid \mathcal{A}_{t}\right)\right\}^{2} \\
& =-E\left(\left\{E\left(\int_{T}^{\bar{T}} r_{s} d s \mid \mathcal{A}_{T}\right)\right\}^{2} \mid \mathcal{A}_{t}\right)+\left\{E\left(\int_{T}^{\bar{T}} r_{s} d s \mid \mathcal{A}_{t}\right)\right\}^{2} \\
& =-V A R\left(E\left(\int_{T}^{\bar{T}} r_{s} d s \mid \mathcal{A}_{T}\right) \mid \mathcal{A}_{t}\right) \\
& =-V A R\left(L \mid \mathcal{A}_{t}\right) .
\end{aligned}
$$

Thus (9.39) becomes

$$
\log K G_{T}(t) / G_{\bar{T}}(t)-\frac{1}{2} V A R\left(L \mid \mathcal{A}_{t}\right)
$$

and, therefore, (9.38) becomes

$$
z_{1}=\frac{1}{\sqrt{V A R(L)}}\left(\log K G_{T}(t) / G_{\bar{T}}(t)-\frac{1}{2} V A R(L)\right) .
$$

Thus

$$
E\left(\mathbf{1}_{Z>z_{1}}\right)=1-N\left(z_{1}\right)=N\left(-z_{1}\right)=N\left(d_{1}\right),
$$


where

$$
d_{1}=\frac{1}{\sqrt{V A R(L)}}\left(\log G_{\bar{T}}(t) / K G_{T}(t)+\frac{1}{2} V A R(L)\right)
$$

as specified in statement of the lemma. The formula for the asset-or-nothing binary put option is derived using call-put parity.

Theorem 3. Let $r_{t}$ be a process for the short rate which satisfies Condition 1. Then the formulae for the expectations $f_{3}$ and $f_{4}$ in (9.1) are given by

$$
\begin{aligned}
& f_{3}(t, T, K, \bar{T})=G_{T}(t) N\left(d_{2}\right) \\
& f_{4}(t, T, K, \bar{T})=G_{T}(t)\left(1-N\left(d_{2}\right)\right),
\end{aligned}
$$

where

$$
\begin{aligned}
d_{2} & =-\frac{1}{2} \sigma_{G}+\frac{1}{\sigma_{G}} \log \frac{G_{\bar{T}}(t)}{G_{T}(t) K} \\
\sigma_{G}^{2} & =V A R\left(E\left(\int_{T}^{\bar{T}} r_{s} d s \mid \mathcal{A}_{T}\right) \mid \mathcal{A}_{t}\right) .
\end{aligned}
$$

Proof. The price of the cash-or-nothing call option on $G_{\bar{T}}$ is

$$
f_{3}(t, T, K, \bar{T})=E\left(\exp \left\{-\int_{t}^{T} r_{s} d s\right\} \mathbf{1}_{G_{\bar{T}}(T)>K} \mid \mathcal{A}_{t}\right) .
$$

We can apply Lemma 9 to the right hand side of (9.48) to give

$$
\begin{aligned}
f_{3}(t, T, K, \bar{T}) & =E\left(\exp \left\{-\int_{t}^{T} r_{s} d s\right\} \mid \mathcal{A}_{t}\right) \times E\left(\mathbf{1}_{L>\log K-C O V\left(-\int_{t}^{T} r_{s} d s, L \mid \mathcal{A}_{t}\right)} \mid \mathcal{A}_{t}\right) \\
& =G_{T}(t) E\left(\mathbf{1}_{Z>z_{2}}\right)
\end{aligned}
$$

for a standard normal random variable $Z$ where

$$
z_{2}=\frac{1}{\sqrt{V A R(L)}}\left(\log K+\operatorname{COV}\left(\int_{t}^{T} r_{s} d s, L \mid \mathcal{A}_{t}\right)-E(L)\right) .
$$

The expression $\log K+C O V\left(\int_{t}^{T} r_{s} d s, L \mid \mathcal{A}_{t}\right)-E(L)$ can be simplified using (9.30) to give

$$
\begin{aligned}
& \log K G_{T}(t) / G_{\bar{T}}(t)+\operatorname{COV}\left(\int_{t}^{T} r_{s} d s, L \mid \mathcal{A}_{t}\right) \\
& +\frac{1}{2} V A R\left(L \mid \mathcal{A}_{t}\right)-\operatorname{COV}\left(L, \int_{t}^{T} r_{s} d s \mid \mathcal{A}_{t}\right) \\
& =\log K G_{T}(t) / G_{\bar{T}}(t)+\frac{1}{2} V A R\left(L \mid \mathcal{A}_{t}\right) .
\end{aligned}
$$

Therefore, (9.50) becomes

$$
z_{2}=\frac{1}{\sqrt{V A R(L)}}\left(\log K G_{T}(t) / G_{\bar{T}}(t)+\frac{1}{2} V A R(L)\right)
$$

and

$$
E\left(\mathbf{1}_{Z>z_{2}}\right)=1-N\left(z_{2}\right)=N\left(-z_{2}\right)=N\left(d_{2}\right)
$$


where

$$
d_{2}=\frac{1}{\sqrt{V A R(L)}}\left(\log G_{\bar{T}}(t) / K G_{T}(t)-\frac{1}{2} V A R(L)\right),
$$

as specified in statement of the lemma. The formula for the cash-or-nothing put option is derived using call-put parity.

Theorem 4. Let $r_{t}$ be a process for the short rate which satisfies Condition 1. Then the formulae for the expectations $f_{5}$ and $f_{6}$ in (9.1) are given by

$$
\begin{aligned}
& f_{5}(t, T, K, \bar{T})=G_{\bar{T}}(t) N\left(d_{1}\right)-K G_{T}(t) N\left(d_{2}\right) \\
& f_{6}(t, T, K, \bar{T})=-G_{\bar{T}}(t)\left(1-N\left(d_{1}\right)\right)+K G_{T}(t)\left(1-N\left(d_{2}\right)\right),
\end{aligned}
$$

where

$$
\begin{aligned}
d_{1} & =\frac{1}{2} \sigma_{G}+\frac{1}{\sigma_{G}} \log \frac{G_{\bar{T}}(t)}{G_{T}(t) K} \\
d_{2} & =-\frac{1}{2} \sigma_{G}+\frac{1}{\sigma_{G}} \log \frac{G_{\bar{T}}(t)}{G_{T}(t) K} \\
\sigma_{G}^{2} & =V A R\left(E\left(\int_{T}^{\bar{T}} r_{s} d s \mid \mathcal{A}_{T}\right) \mid \mathcal{A}_{t}\right) .
\end{aligned}
$$

Proof. Expressing the call option as a combination of an asset-or-nothing call option and a cash-or-nothing call option, we have

$$
f_{5}(t, T, K, \bar{T})=f_{1}(t, T, K, \bar{T})-K f_{3}(t, T, K, \bar{T}) .
$$

Inserting (9.37) and (9.49) into (9.57) gives

$$
\begin{aligned}
c_{T, K, G_{\bar{T}}} & =G_{\bar{T}}(t) E\left(\mathbf{1}_{Z>z_{1}}\right)-K G_{T}(t) E\left(\mathbf{1}_{Z>z_{2}}\right) \\
& =G_{\bar{T}}(t) N\left(-z_{1}\right)-K G_{T}(t) N\left(-z_{2}\right)
\end{aligned}
$$

as required. The formula for the put option is derived from (9.58) and call-put parity.

When the short rate obeys a Vasicek process, the pricing formulae for call and put options on the $\mathrm{ZCB} G_{\bar{T}}$ follow as a corollary of the above theorem.

Theorem 5. Under the Vasicek model, for a strike price $K$ and valuation time $t$, the price of a T-expiry call option on a $\bar{T}$-maturity $Z C B$ is

$$
c_{T, K, G_{\bar{T}}}(t)=G_{\bar{T}}(t) N(h)-K G_{T}(t) N\left(h-\sigma_{G}\right)
$$

and the price of a T-expiry put option on a $\bar{T}$-maturity $Z C B$ is

$$
p_{T, K, G_{\bar{T}}}(t)=-G_{\bar{T}}(t) N(-h)+K G_{T}(t) N\left(-h+\sigma_{G}\right),
$$

where

$$
\begin{gathered}
h=\frac{1}{\sigma_{G}} \log \frac{G_{\bar{T}}(t)}{G_{T}(t) K}+\frac{1}{2} \sigma_{G} \\
\sigma_{G}=\sigma B(T, \bar{T}) \sqrt{\frac{1}{2 \kappa}(1-\exp (-2 \kappa(T-t)))}
\end{gathered}
$$

and

$$
B(t, T)=\left\{\begin{array}{ll}
\frac{1}{\kappa}(1-\exp (-\kappa(T-t))), & \text { if } \kappa>0 \\
T-t, & \text { if } \kappa=0
\end{array} .\right.
$$


Proof. Using Theorem 4 we must compute

$$
\sigma_{G}^{2}=V A R\left(E\left(\int_{T}^{\bar{T}} r_{s} d s \mid \mathcal{A}_{T}\right) \mid \mathcal{A}_{t}\right) .
$$

Firstly, from Lemma 2,

$$
\int_{T}^{\bar{T}} r_{s} d s=r_{T} B(T, \bar{T})+\bar{r}(\bar{T}-T-B(T, \bar{T}))+\sigma \int_{T}^{\bar{T}} B(u, \bar{T}) d Z_{u}
$$

and therefore

$$
E\left(\int_{T}^{\bar{T}} r_{s} d s \mid \mathcal{A}_{T}\right)=r_{T} B(T, \bar{T})+\bar{r}(\bar{T}-T-B(T, \bar{T}))
$$

from which we have

$$
\sigma_{G}^{2}=B(T, \bar{T})^{2} V A R\left(r_{T} \mid \mathcal{A}_{t}\right)
$$

Secondly, from Lemma 1 we have

$$
\operatorname{VAR}\left(r_{T} \mid \mathcal{A}_{t}\right)=\sigma^{2}\left(B(t, T)-\frac{1}{2} \kappa B(t, T)^{2}\right),
$$

which simplifies to

$$
V A R\left(r_{T} \mid \mathcal{A}_{t}\right)=\sigma^{2} \frac{1-\exp (-2 \kappa(T-t))}{2 \kappa} .
$$

It follows that

$$
\sigma_{G}^{2}=B(T, \bar{T})^{2} \sigma^{2} \frac{1-\exp (-2 \kappa(T-t))}{2 \kappa}
$$

and we arrive at the result.

Equation (9.59) agrees with the formula for the price of a call option on a zero coupon bond given in Jamshidian [1989]. However, Jamshidian has made an assumption of risk neutral dynamics and has calculated expectations involving lognormal ZCB prices, omitting many details of the proof, to arrive at the result, whereas we have calculated expectations under the real world measure, giving all details of the proof.

When the short rate obeys a Hull-White process, as in (3.4), the pricing formulae for call and put options on the $\bar{T}$-maturity ZCB follow as a corollary of Theorem 4 .

Theorem 6. Under the Hull-White model, for a strike price $K$ and valuation time $t$, the price of a T-expiry call option on a $\bar{T}$-maturity $Z C B$ is

$$
c_{T, K, G_{\bar{T}}}(t)=G_{\bar{T}}(t) N(h)-K G_{T}(t) N\left(h-\sigma_{G}\right)
$$

and the price of a T-expiry put option on a $\bar{T}$-maturity $Z C B$ is

$$
p_{T, K, G_{\bar{T}}}(t)=-G_{\bar{T}}(t) N(-h)+K G_{T}(t) N\left(-h+\sigma_{G}\right),
$$

where

$$
\begin{gathered}
h=\frac{1}{\sigma_{G}} \log \frac{G_{\bar{T}}(t)}{G_{T}(t) K}+\frac{1}{2} \sigma_{G} \\
\sigma_{G}=B(T, \bar{T}) \sqrt{\int_{t}^{T} \exp \left\{-2 \int_{u}^{T} a(\tau) d \tau\right\} \sigma(u)^{2} d u}
\end{gathered}
$$


FiguRE 6. Comparison of asymptotic formula with Black-Scholes implied option volatilities of options on $10 \mathrm{Y}$ and $20 \mathrm{Y}$ zero coupon bonds based at year 1871 .

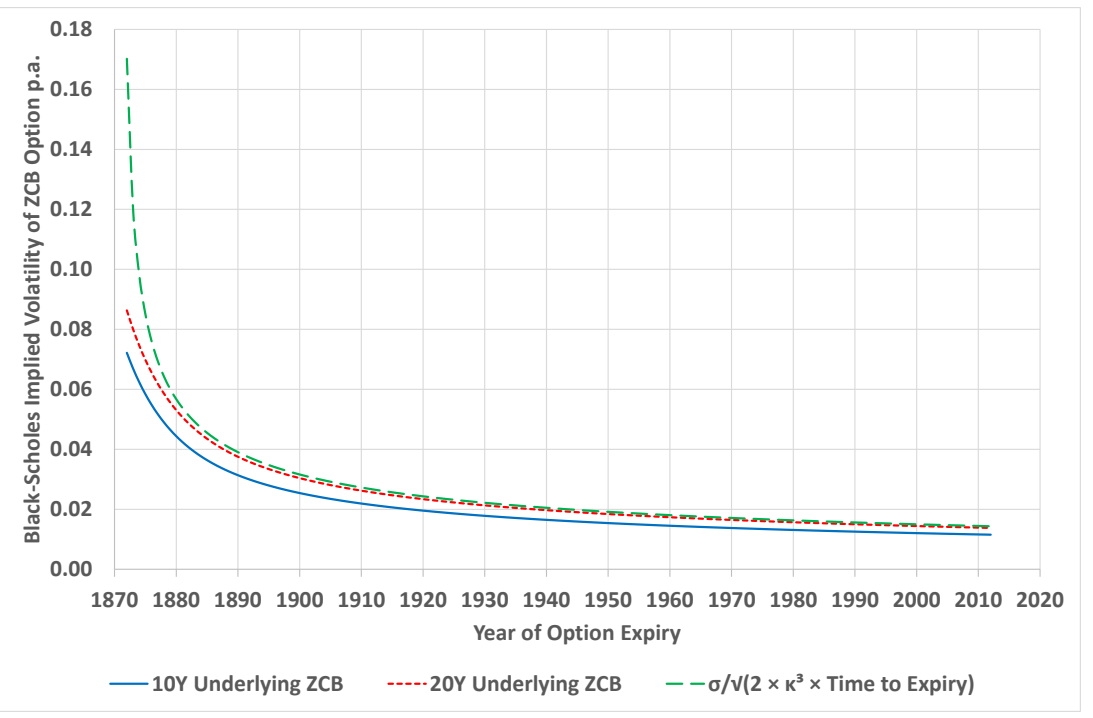

and

$$
B(t, T)=\int_{t}^{T} \exp \left\{-\int_{t}^{s} a(\tau) d \tau\right\} d s .
$$

In Figure 6 the Black-Scholes implied volatilities of options on ten-year and twenty-year ZCBs are shown, based upon the parameters in (5.4). There is good agreement with the theoretical asymptotic formula of the Black-Scholes implied volatility, obtained as

$$
\sigma_{B S}=\frac{\sigma_{G}}{\sqrt{T-t}} \rightarrow \frac{\bar{\sigma}}{\sqrt{2 \bar{a}^{3}(T-t)}}
$$

as $\bar{T} \rightarrow \infty$.

\section{Conclusions}

We have supplied actuarial pricing formulae for zero coupon bonds and call and put options on zero coupon bonds under the Vasicek model, which straightforwardly extend to other Gaussian short rate models. There is scope to generalise these formulae to multifactor Gaussian short rate models in subsequent work. Also, we have shown that extended Vasicek models give rise to sensible long-term bond yields when suitable conditions are imposed on the model parameters. 


\section{REFERENCES}

F. Black and P. Karasinski. Bond and option pricing when short rates are lognormal. Financial Analysts Journal, 47:52-59, 1991.

F. Black, E. Derman, and W. Toy. A one-factor model of interest rates its application to treasury bond options. Financial Analysts Journal, 46:33-39, 1990.

D. Brigo and F. Mercurio. Interest Rate Models - Theory and Practice. Springer Finance, 2001.

D. Brigo and F. Mercurio. Interest Rate Models - Theory and Practice. Springer Finance, second edition, 2006.

J. C. Cox, J. E. Ingersoll, and S. A. Ross. A theory of the term structure of interest rates. Econometrica, 53(2):385-407, 1985.

K. Fergusson and E. Platen. Application of maximum likelihood estimation to stochastic short rate models. Annals of Financial Economics, 10(2), 2015.

C. R. Harvey. The term structure and world economic growth. Journal of Fixed Income, 1:4-47, 1991.

S. Y. Ho and S-B. Lee. Term structure movements and the pricing of interest rates contingent claims. The Journal of Finance, 41:1011-1029, 1986.

J. C. Hull and A. White. Pricing interest rate derivative securities. The Review of Financial Studies, 3(4):573-592, 1990.

F. Jamshidian. An exact bond option formula. The Journal of Finance, 44(1): 205-209, 1989.

E. Platen. A short term interest rate model. Finance and Stochastics, 3:215-225, 1999.

E. Platen. Benchmark model for financial markets. Working Paper, University of Technology, Sydney, 2002.

E. Platen and D. Heath. A Benchmark Approach to Quantitative Finance. Springer Finance, 2006.

E. Platen and D. Taylor. Loading pricing of catastrophe bonds and other longdated, insurance-type contracts. Research Paper 379, University of Technology, Sydney, 2016.

R. Rebonato. Interest-rate option models. Wiley Series in Financial Engineering. John Wiley and Sons, second edition, 1998.

O. A. Vasicek. An equilibrium characterization of the term structure. Journal of Financial Economics, 5:177-188, 1977.

(K. Fergusson) University of Melbourne, Centre for Actuarial Studies, Victoria 3010, Australia

E-mail address, K. Fergusson: kevin.fergusson@unimelb.edu.au 